lIC -426

LA- -11622-MS

Issued: September 1989

DE89 016187

Electron Temperature Measurements of FieldReversed Configuration Plasmas on the FRX-C/LSM Experiment

D. J. Rej

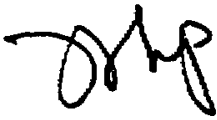

DOCUMENT IS UNLIMATED 


\title{
ELECTRON TEMPERATURE MEASUREMENTS OF FIELD-REVERSED CONFIGURATION PLASMAS ON THE FRX-C/LSM EXPERIMENT
}

by

D. J. Rej

\begin{abstract}
A single-point Thomson scattering cliagnostic is used to determine the electron temperature $T_{e}$ of field-reversed-configuration (FRC) compact toroids generated in the $\theta$-pinch source of the Los Alamos FRX-C/LSM experiment. Measurements are performed close to the axial midplane and near the magnetic and geometric axes. A broad range of plasma conditions is investigated and electron temperatures vary, on average, between 90 and $190 \mathrm{eV}$. For a given condition, $\mathrm{T}_{e}$ remains relatively constant during the $60 \mu \mathrm{s}$ measurement interval between formation and the onset of the $n=2$ rotational instability. $T_{e}$ increases with plasma diameter and external rnagnetic field, and decreases slightly with fill pressure. The temperature at the geometric axis is consistently $10-20 \%$ lower than that near the field null. The temperature is approximately $35 \%$ higher than observed previously on the smaller FRX-C device. The implications of these measurements on plasma confinement properties are discussed.
\end{abstract}

\section{INTRODUCTION AND SUMMARY}

The electron temperature $T_{\theta}$ is an important parameter for magneticallyconfined fusion plasmas. Measurements of $T_{\theta}$ often reveal significant phenomena that contribute to the physics of plasma confinement. The electron temperature has been of special interest in the understanding of the field-reversed configuration, an elongated compact toroid plasma that ideally contains no toroidal magnetic field. Previous measurements have indicated that the electrons are responsible for a substantial amount of power loss in present-day experiments, up to $80 \mathrm{MW}$ or one- 
third of the global loss. ${ }^{1}$ When measured, radiation losses are found to be small, ${ }^{2}$ leading one to speculate that non-classical, cross-field electron thermal conduction dominates. Electron energy confinement is a principal issue that will be addressed in upcoming high-power magnetic compression experiments at Los Alamos. ${ }^{3}$ The influence of the electron temperature on the poloidal flux confinement has been a long-standing issue. The electrical resistivity $\eta$ inferred from flux loss is found to be anomalous, usually ranging between 3 and 20 times the classical Spitzer value; ${ }^{4-6}$ moreover, the classical scaling, $\eta \sim T_{e}^{3 / 2}$, has never been confirmed, indicative of either non-classical resistivities or possible errors in assumptions on the time evolution of the equilibrium current density. ${ }^{7}$

In this report, we present detailed electron temperature data from single-point Thomson scattering measurements of FRCs formed and trapped in situ on the LOS Alamos FRX-C/LSM (LSM) field-reversed $\theta$-pinch. These experiments mark the first comprehensive FRC $\mathrm{T}_{e}$ measurements in over five years. Data have been collected on over 400 discharges during a four-week period beginning in October 1988. Measurements have been made close to the axial midplane and at either of two radii, near the field null or at the geometric axis.

The electron temperature depends on the measurement radius and on the plasma condition. $T_{e}$ values, on average, vary between 90 and $190 \mathrm{eV}$. Both the shot-to-shot variations and the measurement error are typically $10 \%$ to $20 \%$. As in earlier experiments on the smaller FRCs of $F R K-B^{8}$ and $F R X-C,{ }^{1}$ the electron temperature is found to remain relatively constant in time during the period between formation and the onset of the $n=2$ rotational instability.

The electron temperature increases with trapped flux $\phi_{\mathrm{p}}$. With a 3-mtorr initial deuterium fill pressure and a 0.4-T external B-field, the inferred $\phi_{p}$ increases from 1.0 to $7.5 \mathrm{mWb}$ when the reversed bias magnetic field is increased from 37 to $95 \mathrm{mT}$. The plasma separatrix radius $r_{s}$ and $T_{e}$ near the field null increase from $0.11 \mathrm{~m}$ to $0.18 \mathrm{~m}$ and 120 to $190 \mathrm{eV}$, respectively.

There is a weak dependence on the initial fill pressure $p_{o} . T_{e}$ near the field null decreases from 160 to $140 \mathrm{eV}$ when $p_{0}$ is increased from 2 to $4 \mathrm{mtorr}$, while remaining essentially constant at the geometric axis. There is also a weak dependence on the external magnetic field $B_{w}$. $A 10 \%$ increase in $T_{e}$ is observed when $B_{w}$ is increased by $35 \%$.

Linear $\theta$-pinch configurations have been generated without the bias field. Electron temperatures are almost two times smaller than those measured in FRCs, 
consistent with the large thermal conduction losses characteristic of open-field-line configurations.

The temperature at the geometric axis is $7 \%$ to $20 \%$ lower than at the field null. This observation implies a relatively flat $T_{\theta}(r)$ profile inside the separatrix, similar to that found in previous experiments.

When compared to earlier measurements on smaller FRC plasmas produced in $F R X-C,{ }^{1}$ the electron temperature in $L S M$ is found to be higher by approximately $35 \%$. There are no clear indications of increased electron heating during the formation phase on LSM; furthermore, the heating rate during the equilibrium phase after formation has decreased. During equilibrium, the largest source of power input to the electron coritinues to be from classical equilibration with ions, but at substantially reduced rates compared to FRX-C. The higher $\mathrm{T}_{e}$ and the decreased heating imply improved electron energy confinement times on LSM.

The fractional contributions to the global energy confinement have remained essentially unchanged from $F R X-C$. Particle diffusion dominates while the remaining 20 to $40 \%$ of the losses are attributed to nonconvective processes by electrons.

Under the assumption that all nonconvective electron losses are from thermal conduction, transport coefficients are inferred by both dimensional analysis and with a 1\%-dimensional transport code. Even for conditions with optimum confinement, the average cross-field thermal diffusivity is anomalous, $\chi_{\perp e} \simeq 160 \mathrm{~m}^{2} / \mathrm{s}$, which corresponds to 35 times the classical value.

There is no clear correlation between confinement and the electron temperature. In particular, there is no improvement in the inferred poloidal magnetic flux confinement time $\tau_{\phi}$ even when the classical scaling factor $r_{s}^{2} T_{e}^{3 / 2}$ is increased by almost an order of magnitude. Also, no obvious correlations are evident when data are compared with a Bohm-like scaling $\mathrm{BR}^{2} / \mathrm{T}_{\mathrm{e}}$ predicted from low-frequency drift instability theory. ${ }^{9}$

\section{APPARATUS AND EXPERIMENTAL CONDITIONS}

Experiments were performed on the FRX-C/LSM (LSM) facility. ${ }^{10}$ As illustrated in Fig. 1, the front end consisted of a straight 0.70-m-i.d., 2.0-m-long field-reversing $\theta$-pinch coil. Auxiliary "cusp" coils mounted at each end provided a quasi-static Bfield in the direction of the main $\theta$-pinch field; these coils produced end-cusps before field reversal to insure non-tearing formation, and mirror fields to confine the 
FRC after reversal. Plasmas were formed and trapped in the $\theta$-pirinil source. The 0.40-m-i.d., 6.7-m-long stainless-steel translation chamber, however, remained attached to the source during these experiments.

The electron temperature was measured at a single point in space and time on each discharge. The Thomson scattering diagnostic included a Q-switched ruby laser ( $\lambda_{c}=694.3 \mathrm{~nm}, 20-\mathrm{ns}$ pulsewidth, 13-mm diameter, 3-mrad divergence, 5-j output energy) focused from one end to a point $0.2 \mathrm{~m}$ from the axial midplane of the $\theta$-pinch coil and at a radius of either $0.00 \mathrm{sr} 0.10 \mathrm{~m}$ (see Fig. 1). Scattered light was collected, dispersed and detected with a 7-channel, triple-grating polychromator similar to the one used on the Scylla-INP $\theta$-pinch experiment. ${ }^{11}$ The polychromator was configured to detect light wavelengths between 658 and $692 \mathrm{~nm}$. Photomultiplier currents were measured with gated analog-to-digital converters (LeCroy modei 2249). Plasma background signals were recorded $100 \mathrm{~ns}$ before and $100 \mathrm{~ns}$ after the laser puise. Data were processed and $\mathrm{T}_{\mathrm{e}}$ was determined immediately after each discharge with a Prime model 750 computer. Data from an LSM discharge are plotted in Fig. 2.

Other "on-line" diagnostics operational during this data run included an excluded-flux array used to measure the axial profile of the separatrix radius $r_{s}(z)$, and a single-chord interferometer aligned to measure $\int \mathrm{ndl}$ across the diameter at the axial midplane $z=0$. In addition, a new 7-chord instrument positioned at $z=0$ was being checked out during a portion of this data run. An image-intensifying streak camera was positioned side-on to monitor radial motion of the plasma column near the axial midplane. Two ion temperature diagnostics, a polychromator tuned to detect the 227.1-nm CV line and a time-integrated neutron detector, were also installed on the experiment.

Electron temperatures were measured at both radial positions during the time interval $10 \leq t \leq 70 \mu \mathrm{s}$ between FRC formation and the onset of the $n=2$ instability which usually terminated the discharge. A variety of plasma conditions were produced by adjusting three external parameters: (1) the initial deuterium fill pressure $p_{o}$; (2) the reversed bias magnetic field $B_{b}$; and (3) the external equilibrium magnetic field $B_{w}$. The fill-pressure scan was performed at $B_{b} \simeq 60 \mathrm{mT}$ and $B_{w} \approx 0.4 T$ (at time $t=30 \mu \mathrm{s}$ ) with $p_{o}$ set at either 2, 3, 4 or 5 mtorr. The bias-field scan, $37 \leq \mathrm{B}_{\mathrm{b}} \leq 95 \mathrm{mT}$, was performed at 3 mtorr and $\mathrm{B}_{\mathrm{w}}=0.4 \mathrm{~T}$. There were also limited measurements at 5 mtorr and large bias, $B_{b}=100 \mathrm{mT}$, a condition where in earlier experiments a soft $x$-ray diagnostic revealed large flute-like 
distortions of the plasma column. ${ }^{6} \mathbf{B}_{\mathbf{w}^{\prime}}$ could be varied by adjusting the capacitance of the main $\theta$-pinch bank. At $p_{o}=3$ mtorr and $B_{b}=65 m T, B_{w}$ was increased from $0.4 \mathrm{~T}$ to $0.6 \mathrm{~T}$.

\section{EXPERIMENTAL OBSERVATIONS}

\section{A. Fill Pressure Scan}

As the fill pressure was increased from 2 to 5 mtorr, with bias and external Bfields kept constant, colder, higher-density FRCs were produced. The experimental conditions and the plasma parameters are listed in Table I; each value represents an average of many discharges, while its uncertainty denotes the standard deviation. For each fill pressure, the bias magnetic field and the timing between the $\theta$-pinch preionization $(\mathrm{Pl})$ and main capacitor bank discharge were tuned to values that previously led to optimized confinement times. ${ }^{6}$ The plasma parameters quoted in Table I were measured at time $\mathrm{t}=30 \mu \mathrm{s}$, while the time-averaged flux, energy, and particle confinement times $\left(\tau_{\phi}, \tau_{E}\right.$, and $\left.\tau_{N}\right)$ were obtained with a $0-D$ transport model. 5,12 For all fill pressures, the separatrix radius $r_{s}$ remained relatively constant at $0.16 \pm 0.01 \mathrm{~m}$. The corresponding field-null radius, $R \simeq 0.11 \mathrm{~m}$, was inferred from the equilibrium relationship, $R=r_{3} / \sqrt{2}$, derived for an elongated FRC. ${ }^{8}$ [Note: For the remainder of this report, the $T_{e}$ measurements performed at $r=0.10 \mathrm{~m}$ will be quoted as that near the field null $r \approx R$.] The FRC confinement times were sufficiently long for most fill pressures, $p_{0} \leq 4 \mathrm{mtorr}$, for one to confidently believe that an FRC equilibrium existed. At 5 mtorr, however, the short confinement times cast some doubt on the true nature of the plasma configuration. Further discussion of the FRC confinement properties can be found in Section IV.

Electron temperatures at each fill pressure and radial position are plotted against time in Figures 3 through 6. The main $\theta$-pinch capacitor bank was discharged at time $t=0$. Each data point corresponds to a separate discharge while its error bar denotes the uncertainty in the Gaussian fit. For all cases except 5 mtorr, a handful of shots with very poor confinement, $\tau_{\phi}<75 \mu \mathrm{s}$, have been excluded from these $T_{\theta}(t)$ plots.

To within the experimental error and shot-to-shot scatter, the electron temperature remained constant in time. The time and shot averaged temperatures for each condition are listed in Table II. In computing the average $T_{e}$, equal weight 
was given to each data point, regardless of the error in the Gaussian fit. The uncertainties quoted in Table II correspond to the standard deviation of the computed average. For reference, an average ion temperature $T_{1}$ at $t=30 \mu s$ is quoted. $T_{1}$ was obtained from the difference between pressure balance temperature at $t=30 \mu$ s and the field-null $T_{e}$.

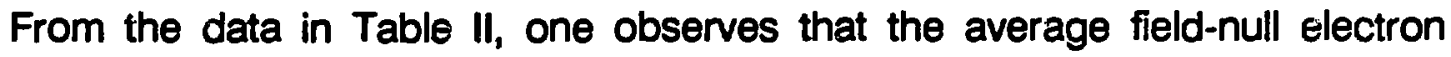
temperature decreased by $15 \%$ when the fill pressure was increased from 2 to 4 mtorr. $T_{\theta}$ at the geometric axis, on the other hand, remained relatively constant at $133 \mathrm{eV}$. For all cases except $p_{0}=5 \mathrm{mtorr}$, the inferred ion temperature at $r=R$ and $\mathrm{t}=30 \mu \mathrm{s}$ was 2 to 3 times larger $\operatorname{than} \mathrm{T}_{\boldsymbol{e}}$.

\section{B. Blas Field Scan}

The most extensive reversed-bias-field scan was performed at $p_{o}=3$ mtorr, with $\mathrm{B}_{w}=0.41 \pm 0.04 \mathrm{~T}$, and $\mathrm{PI}$ timing of $18.6 \pm 0.1 \mu \mathrm{s}$. The bias field $\mathrm{B}_{\mathrm{b}}$ was varied between 37 and $95 \mathrm{mT}$, an operation range where reasonably-long configuration lifetimes were observed. The electron temperature was measured immediately after formation of the FRC equilibrium, at time $t=30 \mu \mathrm{s}$.

As illustrated in Figure 7, when the bias flux was increased, more poloidal flux was measured both at field reversal and in the FRC equilibrium. Typically, 35 to $65 \%$ of the initial bias flux remained accounted for during the "zero crossing" (i.e., at time $t=0$ when the magnetic field reverses at the wall). Approximately 30 to $60 \%$ of the zero-crossing flux was trapped in the equilibrium FRC, consistent with the predictions of a semiempirical formation model. ${ }^{13}$ Consequently, the FRC separatrix radius increased with $B_{b}$ from 0.10 to $0.19 \mathrm{~m}$ (see Fig. 8). Other plasma parameters also varied. As indicated by the data points in Figure 9 and from the shot averages in Table III, larger-diameter plasmas were hotter, less dense, shorter, and contained a larger particle inventory (up to $80 \%$ of the initial fill) at $t=30 \mu \mathrm{s}$.

The electron temperature, on average, also increased with the plasma diameter. The data plotted in Figure 10 reveal a $90 \%$ increase in the upper bound of $T_{e}(r=R)$ when $r_{s}$ was increased by $60 \%$. Unweighted shot averages of the same data are plotted in Figure 11. Logarithmic least-squares fits to these data yielded the empirical scaling,

$$
T_{\theta}(r \simeq R) \simeq 13 r_{s}^{0.92}
$$




$$
T_{\theta}(r=0) \approx 10 r_{s}^{0.94},
$$

where $T_{\theta}$ is in $\theta V$ and $r_{s}$ is in $\mathrm{cm}$. The inferred ion temperature at $t=30 \mu s$ did not change significantly, as illustrated by the data in Figure 12; however, the average $T_{i}$ showed the weak empirical scaling,

$$
T_{1}(r=R)=143 r_{s}^{0.33}
$$

A few discharges were made at higher bias fields, $B_{b}=96 \mathrm{mT}$, conditions where poor confinement $\left(\tau_{\phi} \leq 60 \mu \mathrm{s}\right)$ was consistently observed. The measured temperature, $T_{\theta}=175 \mathrm{eV}$, did not differ discernibly from that observed in the betterconfined FRCs at lower bias field.

A few high-bias oilscharges were obtained at 5-mtorr fill pressure, conditions where the soft $x$-ray diagnostic revealed high toroidal mode number perturbations at the plasma edge. For $B_{b}=92 \mathrm{mT}$, the typical plasma parameters at $30 \mu \mathrm{s}$ were $B_{w} \approx 0.4 \mathrm{~T}, r_{s} \simeq 0.17 \mathrm{~m}, \phi_{p} \approx 5 \mathrm{mWb},<n>\approx 9 \times 10^{20} \mathrm{~m}^{-3}, \mathrm{~T}_{\mathrm{e}}+\mathrm{T}_{i} \approx 400 \mathrm{eV}$. The confinement times were poor: $\tau_{\phi} \simeq 31 \mu \mathrm{S}, \tau_{N} \simeq 52 \mu \mathrm{S}, \tau_{E} \simeq 27 \mu \mathrm{s}$. The average electron temperatures were $128 \pm 12 \mathrm{eV}$ at $r \approx R$ and $118 \pm 16 \mathrm{eV}$ at $r=0$. The $T_{e}$ values were not appreciably different from the low-bias case (see Table I) but larger than those of open-field-line plasmas (see below).

\section{Equilibrium Field Scan}

To date, the best flux trapping and confinement parameters on LSM were obtained at reduced plasma energy densities and external field $\mathrm{B}_{\mathrm{w}}$. The data presented in the above sections were obtained at these "degraded" conditions. In this section, we present results obtained with larger B-fields corresponding to the operation of the entire main $\theta$-pinch capacitor bank. Experiments were performed at 3-mtorr fill pressure and modest bias fields, $B_{b}=65 \mathrm{mT}$. The changes in FRC parameters that resulted from the $40 \%$ increase in $B_{w}$ are listed in Table IV. In general, the higher-field plasmas were smaller, more dense, hotter, contained less flux, and had poorer confiriement. The density and pressure-balance temperature increased as expected from the adiabatic compression theory scaling, ${ }^{14} n \sim B_{w} 6 / 5$ and $T-B_{w}{ }^{4 / 5}$. 
The electron temperature showed a modest increase with $B_{w} . T_{e}$ at $r \approx R$ and $r=0$ for the high-field case are plotted in Figure 13. As observed at low $B_{w}$ the temperature remained constant in time, to within the scatter. Shot and timeaveraged temperatures are quoted in Table $\mathrm{V}$. The average $\mathrm{T}_{e}$ increased by 10 to $15 \%$ which was less than both the standard deviation (20\%) and the prediction from adiabatic theory (27\%).

A noteworthy feature of the data at $r=0$ in Figure 13 is the large magnitude of the error bars, which usually exceeded the shot-to-shot scatter in $T_{e}$. The fitting error for this case was consistently larger than for every other condition investigated; however, there were systematic features. As illustraced by the representative data in Figure 14, the scattered signal consistently deviated from the expected Gaussian, appearing more like a two-temperature distribution.

\section{Open-Field Configurations}

It is interesting to compare the FRC with the linear $\theta$-pinch configuration. These open-field-line configurations were generated on LSM by operating without the reversed bias field. During the Thomson scattering run at $r=0$, with $B_{w}=0.33$ to $0.43 \mathrm{~T}$ and $p_{0}=3$ and 4 intorr, a few linear $\theta$-pinch discharges were made. For all cases, a small plasma column with an excluded filux radius of $0.10 \mathrm{~m}$ formed and decayed away rapidly, typically in $30 \mu \mathrm{s}$. The electron temperature of $85 \pm 10 \mathrm{eV}$ was consistently less than that in an FRC formed with similar PI and $\mathrm{B}_{w}$.

\section{HEATING AND CONFINEMENT}

In this section, the Thomson scattering data are interpreted. Power flow in the electron channel is studied and transport coeificients are estimated by both dimensional analysis and with a 1\%-dimensional transport code. The contributions of electron losses to the global power balance are assessed. The effect of the electron temperature on the poloidal flux confinement is also considered.

When compared to the smaller FRCs produced in FRX-C, the electron temperature in LSM is about 35\% higher. For example, the plasma density and magnetic field produced at $4 \mathrm{mtorr}$ in $L S M, n=1.1 \times 10^{21} \mathrm{~m}^{-3}$ and $0.45 \mathrm{~T}$ with $r_{s}=0.17 \mathrm{~m}$ (see Tables ! and II), are virtually identical to those measured on FRX-C at "degraded" 5 -mtorr conditions with $r_{s} \approx 0.12 \mathrm{~m}$. ${ }^{15}$ The electron temperature near 
the field null is $140 \mathrm{eV}$ on LSM and only $105 \mathrm{eV}$ on FRX-C. There exist two plausible reasons for the higher electron temperature: (1) more heating; and/or (2) better confinement. These explanations are further explored in the analysis below.

\section{A. Electron Heating}

Electron heating occurs in two distinct phases, during and after FRC formation. A semi-empirical formation model ${ }^{13}$ has been used to estimate the heating source terms. Given the size of the liftoff flux, the azimuthal elestric field, and the fill pressure, this model can predict the FRC total temperature $T$ after formation; moreover, it can estimate the relative magnitudes of two different sets of heating mechanisms: (1) radial shock and compression; and (2) the combination of axial shock and resistive annihilation of reversed magnetic flux. As an example, we compare the same 4-mtorr LSM and degraded 5-mtorr FRX-C cases considered in the preceding paragraph. As demonstrated in Ref. 13, the model correctly predicts the inferred temperature, $T \simeq 430 \mathrm{eV}$. On $F R X-C$, resistive and axial heating appears to be equally important as radial heating. For the 4-mtorr LSM case, radial heating dominates, accounting for $75 \%$ of the plasma energy. The difference between the two experiments can be attributed to a higher normalized liftoff flux, $G_{L O}=0.28$ on FRX-C as compared with 0.17 on LSM. Unfortunately, the formation model does not predict how the formation energy is divided between electrons and ions. While there is no theoretical basis for more electron heating on LSM, one might speculate that there should be less. Classically, one expects most of the shock energy to be deposited to the ions, resistive heating to the electrons, and the compression heating given by the adiabatic laws subject to initial conditions and ion-electron equipartition. In this "classical" context, one would expect a higher $T_{e}$ on FRX-C and a higher $T_{1}$ on LSM. These expectations are contrary to observations, indicating that the higher temperatures on $L S M$ cannot be explained by increased heating during formation; however, this conclusion is weak since the classical picture is probably not a realistic description of the turbulent FRC formation process.

It is somewhat easier to understand FRC energy balance during the decay phase after formation. Electrons are heated by at least three sources: (1) Coulomb collisions with hotter ions; (2) magnetic compression; and (3) ohmic heating. The electron heating rate due to Coulomb collisions with ions is 


$$
d T_{0} / d t=\left(T_{1}-T_{0}\right) / T_{\iota q},
$$

with the energy equilibration time between deuterons and electrons, ${ }^{16}$ $\tau_{e q}=6.3 \times 10^{14} \mathrm{~T}_{\bullet}^{3 / 2} / \mathrm{n} \mathrm{InA_{ej }}$ [units: $\mu \mathrm{s}, \mathrm{eV}$, and $\mathrm{cm}^{-3}$ ], where $\mathrm{In \Lambda}$ is the Coulomb logarithm which is about 12 for LSM. The equilibration time near the field null typically ranges from 80 to $200 \mu s$, depending on conditions. The average equilibration heating rates at $r \approx R$ computed from Eq. 1 are listed in Table VI. Comparing FRCs with comparable density and B-field, one finds that the power flow from electron-ion equilibration on LSM is more than two times smaller than on FRXC. Nevertheless, equilibration usually accounts for most of the input energy to the electrons. Values range from $1.2 \mathrm{eV} / \mathrm{\mu s}$ at 3 mtorr, large bias, and low external field, to $3.4 \mathrm{eV} / \mu \mathrm{s}$ at low bias and high field. The relatively large standard deviations reflect the shot-to-shot variations in $\mathrm{T}_{\theta}^{3 / 2}$.

The power to electrons from adiabatic magnetic compression can be estimated by the equation ${ }^{14}$

$$
d T_{\theta} / d t=2 T_{\theta} / 3 T_{v}
$$

where $\tau_{v}=-V_{s} /\left(d V_{s} / d t\right)$ is the characteristic decay time of the separatrix volume $v_{s}$. Typically, $\tau_{v}$ varies between 2 and 4 times the global energy confinement time $\tau_{E}$. The compression heating rate ranges between 0.3 and $2 \mathrm{eV} / \mathrm{ils}$, depending on conditions (see Table VI). For all cases except $p_{0}=5$ mtorr, the compression power is relatively small, less than one-half of the equilibration input power.

The ohmic power input to the plasma is given by the relationship ${ }^{5}$

$$
d\left(T_{0}+T_{1}\right) / d t=\left(T_{0}+T_{i}\right) / \tau_{\Omega},
$$

where $\tau_{\Omega}=3<\beta>\tau_{\phi} / 4(1-<\beta>)$. From Eq. 3 , the ohmic heating rate near the field null is estimated at 0.5 to $2 \mathrm{eV} / \mu \mathrm{s}$. Because of the anomalous resistivity inferred from flux loss (see below), a sizable fraction of this power can go to the ions. As done previously, ${ }^{5}$ the fraction to electrons is assumed to be $0.3 \times\left(1+T_{e} / T_{i}\right)$ $(\approx 42 \%$ on LSM), consistent with lower-hybrid-drift turbulence. Values based on 
this model are listed in Table VI. The ohmic heating rate to electrons at $r \approx R$ is typically 0.2 to $0.3 \mathrm{eV} / \mu \mathrm{s}$; larger rates are inferred for conditions with short $\tau_{\phi}$ (5 mtorr) or large $\phi_{p}$ (3 mtorr, high bias).

\section{B. Electron Energy Confinement}

During the equilibrium phase after formation, the electron energy oalance is given by the equation

$$
(3 / 2) d\left(N T_{\theta}\right) / d t=P_{\text {in }}-P_{N_{\theta}}-P_{E_{\theta}}
$$

where $\mathbf{N}$ is the electron inventory inside the separatrix, and $P_{\text {In }}$ represents the integrated input power to the electrons due to ion-electron equilibration, compression, and ohmic dissipation, while $P_{\mathrm{Ne}_{\theta}}$ and $P_{\mathrm{E}_{\theta}}$ represent losses due to particle diffusion and "nonconvective" processes (e.g., thermal conduction, ionization, and/or radiation), respectively. Electrons diffuse across field lines and escape from the system, each carrying away an energy $(5 / 2) \mathrm{T}_{e}{ }^{; 17}$ therefore, the "convective" electron power loss cari be estimated by the equation

$$
P_{\mathrm{Ne}} \approx(5 / 2) N T_{\theta} / \tau_{\mathrm{N}}
$$

After substituting the relationship, $d N / d t=-N / \tau_{N}$, Eq. 4 reduces to a simpler form,

$$
(3 / 2) N\left(d T_{\theta} / d t\right)=P_{\text {in }}-N T_{\theta} / T_{H}-P_{E_{\theta}}
$$

In our analysis, we restrict ourselves to low-bias conditions where the electron temperature has been measured against time (cf. Figs. 3-5 and 13) and found to be relatively constant, l.e., $\mathrm{dT} / \mathrm{dt} \approx 0$. The average powers $P_{\text {in }}, P_{\mathrm{Ne}}$, and $P_{\mathrm{Ee}}$ for these conditions are listed in Table VIll. $P_{\text {in }}$ is found by integrating and summing the electron heating rates of Table VI. $P_{\mathrm{Ne}}$ is computed from Eq. 5 with the experimental $\tau_{N}$ determined from the $D-D$ analysis of interferometer and excluded flux data. $P_{E \theta}$ is then computed from the difference $P_{\text {in }}-N T_{e} / \tau_{N}$. 
From the data in Table VII, one finds that $P_{\text {In }}$ is substantial, ranging between 40 and $80 \mathrm{MW}$. The nonconvective and convective electron power losses are comparable. The losses have been also compared to the total power loss from the plasma, $P_{\text {tot }}=(3 / 2) N\left(T_{\theta}+T_{p}\right) / T_{E}$, which is determined from $0-D$ analysis of the excluded flux data. $P_{E \theta}$ ranges from $21 \%$ of $P_{\text {tot }}$ at 2 mtorr to $38 \%$ at $4 \mathrm{mtorr}$. As in earlier studies, particle diffusion still dominates the global energy confinement. The total convective power loss from electrons and ions, $P_{\mathrm{Ne}_{e}} \times\left(1+T_{i} / T_{\theta}\right)$, accounts for at least $60 \%$ of $P_{\text {tot }}$ in all cases.

In the experimental limit where $d T_{\theta} / d t=0$, the nonconvective electron energy confinement time $\tau_{E \theta}$ is given by the relationship, $\tau_{E \theta} \approx(3 / 2) N T_{e} / P_{E e}$. From the data in Table VII, $\tau_{E e}$ is estimated to be approximately $100 \mu s$ for the 2 and 3 mtorr cases and $65 \mu \mathrm{s}$ for 4 mejrr. These confinement times are better than on FRX-C from which $\tau_{E_{e}}$ values as low as $33 \mu$ s can be inferred. ${ }^{5}$

A variety of effects might be responsible for the large nonconvective electron power loss, $\mathbf{P}_{\mathrm{Ee}_{\boldsymbol{\theta}}}$. Possible candidates are ionization, radiation, or thermal condisction. Ionization losses have never been measured in an FRC but have been estimated to be small, ${ }^{18}$ in view of the comparatively low neutral flux and very short mean free path in the sheath plasma outside the separatrix. Radiation losses have not been measured on LSM; however, earlier bolometer measurements ${ }^{2}$ on FRX$C / T$ indicated that radiative losses are small, accounting for $7 \%$ of $P_{\text {tor }}$. By the process of elimination but without experimental verification, the relatively large magnitude of $\mathrm{P}_{\mathrm{Ee}}$ is often attributed to cross-field thermal conduction.

We now further pursue the speculation that $P_{E_{\theta}}$ is entirely due to electron thermal conduction radially outwards across magnetic field lines. Dimensional analyses in the paragraphs below are followed by a more detailed computation with the 1/4-D transport code in Section IV.D. Previous Thomson scattering measurements on $F R X-C$ indicated that the electron temperature at the separatrix is the same as at $r=0$, consistent with our expectation that $T_{e}$ is constant on flux surfaces; therefore, for the remainder of this report, we will assume separatrix temperatures equal to those actually measured at the geometric axis.

The electron heat flux $q$ due to thermal conduction for the elongated FRC is given by the equation

$$
q=\kappa_{\perp} d T_{e} / d r
$$


A crude estimate of the electron cross-field thermal conductivity $\kappa_{\perp}$ can be made from a dimensional analysis. Assuming that the nonconvective power loss is uniformly distributed over the separatrix area, $A_{s}=\pi r_{s}^{2} I_{s}$, and that there is a linear temperature profile across the minor radius, $a=r_{s}-R$, one can rewrite Eq. 7 as

$$
\kappa_{\perp} \simeq P_{E \theta} a / \Delta T_{\theta} A_{s}
$$

where $\Delta T_{\theta}=T_{\theta}(R)-T_{\theta}\left(r_{s}\right)$. The $\kappa_{\perp}$ values predicted by $E q .8$ range from $1.6 \times 10^{23} \mathrm{~m}^{-1} \mathrm{~s}^{-1}$ at 2 mtorr to $7.5 \times 10^{23}$ at $4 \mathrm{mtorr}$ (see Table VIII). The corresponding perpendicular electron thermal diffusivity, $x_{\perp}=\kappa_{\perp} / n$, varies between 250 and $715 \mathrm{~m}^{2} \mathrm{~s}^{-1}$.

The inferred electron thermal conductivity can be compared to the classical value $\kappa_{\perp}$, which is given by ${ }^{16}$

$$
\kappa_{\perp c}=4.7 n T_{\theta} / m_{\theta} \omega_{c e}{ }^{2} \tau_{\theta \theta}
$$

Using the average measured values of $n$ and $T_{\theta}$ and the average $B$ between $R$ and $r_{s}$ computed from the rigid-rotor equilibrium profile, $\kappa_{\perp c}$, has been computed using Eq. 9 and found to be significantly smaller than the above experimental values (see Table VIII). $\kappa_{\perp} / \kappa_{\perp c}$ varies from 54 at $3 \mathrm{mtorr}$ and low field to 140 at $4 \mathrm{mtorr}$. These numbers may be compared to earlier FRX-C measurements ${ }^{1}$ at full-field 5 mtorr, where $n \approx 1.9 \times 10^{21} \mathrm{~m}^{-3}, B_{w} \approx 0.8 \mathrm{~T}, r_{s} \approx 0.10 \mathrm{~m}, \mathrm{~T}_{e}(R) \simeq 175 \mathrm{eV}, \mathrm{T}_{\mathrm{e}}\left(\mathrm{r}_{\mathrm{s}}\right) \approx$ $150 \mathrm{eV}$, and $\kappa_{\perp} / \kappa_{\perp c} \simeq 80$. While anomalous, the inferred $\kappa_{\perp}$ remains four orders of magnitude smaller than the classical value along magnetic field lines, $\kappa_{\| l}=$ $3.2 \times n T_{e} \tau_{\theta e} / m_{e}$ (see Table VIII).

The LSM $\chi_{\perp}$ values may at first seem large, especially when they are compared to those obtained from other plasma configurations. For example, $x_{\perp}$ typically ranges between 0.2 and $10 \mathrm{~m}^{2} \mathrm{~s}^{-1}$ in tokamaks. ${ }^{19}$ it should be noted, however, that the classical diffusivities in these higher-field, less-collisional devices are significantly smaller than for the FRC; consequently, the anomaly factors for tokamaks are enormous. As an example, we consider ohmic discharges in the JET tokamak, in which a minimum $x_{\perp}$ of $0.2 \mathrm{~m}^{2} \mathrm{~s}^{-1}$ was reported. ${ }^{20}$ For discharges where $\mathrm{T}_{\theta} \simeq 5 \mathrm{keV}, \mathrm{n} \approx 3 \times 10^{19} \mathrm{~m}^{-3}$, and $\mathrm{B} \simeq 2.8 \mathrm{~T}$, the classical thermal diffusivity is estimated to be $6 \times 10^{-4} \mathrm{~m}^{2} \mathrm{~s}^{-1}$. The corresponding thermal conduction anomaly 
factor is over 300 . This anomaly grows by more than an order of magnitude for auxiliary-heated discharges on JET, where $\chi_{\perp}$ values of $2.5 \mathrm{~m}^{2} \mathrm{~s}^{-1}$ were reported. ${ }^{21}$

The FRC electron temperature is approximately twice that measured in openfield-line configurations (cf. Section III.D). The heating terms for the equilibrium FRC are no greater than for the open configuration; therefore, one can conclude that the closed field lines improve the electron energy confinement. The measured $\mathrm{T}_{e}$ values for the FRC are consistently larger than the predictions of two theories based on open-field-line electron energy confinement. The first theory is that of Newton, ${ }^{22}$ who has computed the edge $\mathrm{T}_{e}$ for an elongated, sharp-boundary $F R C$ in the limit where ohmic dissipation in the sheath is balanced by classical electron thermal conduction along open field lines to the end walls; furthermore, perpendicular particle flow across the sheath is balanced against parallel free-streaming ion losses. The resulting electron temperature is independent of the particular resistivity model and is given by the equation

$$
T_{e}=5 \times B^{2 / 3} L^{1 / 3},
$$

where $T_{\theta}$ is in eV, the magnetic field $B$ is in $k G$, and the coil length $L$ is in $\mathrm{cm}$. The model assumes collisional electrons on open field lines, which is reasonable for LSM since $\lambda_{e \theta} / L$ in the sheath is typically between 0.05 and 0.1 . Substituting the experimental parameters into Eq. 10, the Newton temperature ranges from approximately $86 \mathrm{eV}$ for low-field conditions to $103 \mathrm{eV}$ at high field. These values are consistently $1 / 3$ lower than the experimental values inferred at $r=r_{s}$; however, excellent agreement is obtained between the Newton temperature and that measured in the open-field configurations.

The second theory has been published by Correll, ${ }^{23}$ who computed a limiting electron temperature for the situation where the power from classical equilibration between hot ions and cold electrons is balanced by electron thermal conduction and convection along open magnetic fields. In the collisional limit where conduction dominates, the limiting temperature is given by an equation which has a form remarkably similar to Eq. 10 ,

$$
T_{\theta}=0.22 \times\left[\beta_{i} B^{2} L^{2} / \lambda_{\theta \theta}\right]^{1 / 3},
$$


where $\beta_{1}=\beta T_{1} /\left(T_{e}+T_{i}\right)$ and the units are $\mathrm{eV}, \mathrm{kG}$, and $\mathrm{cm}$. Using average sheath plasma parameters, $n \simeq\langle n\rangle / 2$ and $T_{e} \approx T_{e}\left(r_{s}\right) / 2$, Equation 11 predicts temperatures ranging from $85 \mathrm{eV}$ for the low-field, low-bias 2 and 3 mtorr cases to about $105 \mathrm{eV}$ for both $4 \mathrm{mtorr}$ and high-field $3 \mathrm{mtorr}$. For FRC parameters inside the separatrix, Eq. 11 predicts temperatures which are 20 to $30 \%$ lower than those computed for the sheath.

An important experimental observation is the dependence of $T_{e}$ on the plasma radius $R$. As mentioned above, $T_{\theta}$ increases almost proportional with $R$; moreover, for virtually identical $n, B$ and $L$ the larger LSM plasrinas are $35 \%$ hotter than on FRX$C$. This scaling with radius is inconsistent with both the Newton and Correll theories. We conclude that the two theories are useful in estimating an average temperature of the open-field-line sheath outside the separatrix, since they consistently predict temperatures 60 to $80 \%$ of the experimental value at $r=r_{s}$; however, these predictions are only lower bounds for the FRC core temperatures for the limit of no closed-field-line confinement. According to the theories, the electron temperature should increase in higher-field experiments. For example, for the LSM Compressor ${ }^{3}$ where $B=20 \mathrm{kG}$ and $\mathrm{L} \approx 350 \mathrm{~cm}$, Eqs. 10 and 11 both predict a minimum electron temperature of about $275 \mathrm{eV}$. If confinement in the closed-field region continues to be better than on open lines, the average sheath temperature will remain at $50-70 \%$ of the FRC core; therefore, record $T_{\epsilon}(R)$ values of 400 to $550 \mathrm{eV}$ could be likely.

\section{Flux Confinement}

The characteristic decay time of the poloidal magnetic flux, $\tau_{\phi}=-\phi_{p} /\left(d \phi_{p} / d t\right)$, can be written as a combination of Faraday's and Ohm's laws at the field null, which for the rigid-rotor equilibrium profile is 5

$$
\tau_{\phi}=7.4 \times 10^{-8} r_{s}^{2} / \eta(R)
$$

where $\eta(R)$ is the resistivity at the field null and all units are in mks. For each discharge, $\tau_{\phi}$ is inferred from the decay of the excluded flux, thereby allowing one to estimate $\eta(R)$ from Eq. 12.

Traditionally, the inferred field-null resistivity has been compared to the classical, cross-field Spitzer value $\eta_{\perp c}$, even though $\eta(R)$ is always found to be anomalous 
and the classical scaling is never observed. With $Z_{\text {eff }}=1$ and $\ln \Lambda_{e l}=12$, the classical resistivity is given by ${ }^{16}$

$$
\eta_{\perp c}=1.2 \times 10^{-3} / T_{e}^{3 / 2}
$$

$\Omega$-m, where $T_{e}$ is in $\theta V$. Using the electron temperatures measured near the field null, we have computed $\eta_{\perp c}(R)$ with Eq. 13 for each discharge; subsequently, the classical flux confinement time $\tau_{\phi c}$ is determined with Eq. 12, and compared to the actual $\tau_{\phi}$ inferred from excluded flux. The inferred resistivity anomaly factor, $\eta(R) / \eta_{\perp c}(R) \approx \tau_{\phi c} / \tau_{\phi}$, is always greater than 8 , and can be as large as 150 under certain conditions. Data from the 3-mtorr, bias-field scan (see Table III and Figs. 7-12) offer an excellent opportunity to compare $\tau_{\phi}$ with classical losses, since the classical scaling factor, $r_{s}{ }^{2} T_{e}{ }^{3 / 2}$, varies by almost an order of magnitude. As illustrated by the data in Fig. 15a, there is no clear correlation between $\tau_{\phi}$ and $\tau_{\phi c}$, indicative of a resistivity anomaly factor that increases from about 10 at low bias to 100 at high bias. Ignoring equilibrium changes (see below), one must conclude that the field-null resistivity or flux-loss anomaly on LSM is higher than on FRX-C where anomaly factors between 3 and 7 were reported. 4,5

The inferred flux loss remains poorly uniderstood at present. This fact is compounded by recent 2-D MHD equilibrium computations ${ }^{7}$ which indicate that the $\tau_{\phi}$ inferred in experiments could be subject to sizable errors. These errors result from variations in the current density at the field null that could go undetected by the usual excluded flux diagnostic. Unfortunately, there is no good way at present to estimate the magnitude of possible equilibrium changes.

A new theory, ${ }^{9}$ based on turbulence from low-frequency-drift (LFD) instabilities, has been recently developed to explain observed flux and particle loss in an FRC. A "crude" estimate of the flux confinement time from LFD activity has been derived,

$$
\tau_{\phi} \simeq 3 \times 10^{-8} \mathrm{Br}_{s}^{2} / T_{e}\left[1+T_{e} / T_{i}\right]^{1 / 2}
$$

where $\tau_{\phi}$ is given in seconds, $B$ in gauss, temperature in $\theta V$, and $r_{s}$ in $\mathrm{cm}$. The particle confinement time $\tau_{N}$ is given by the same equation. The flux confinement data from the 3-mtorr bias scan have been compared to the theoretical values predicted from Eq. 14. Results are plotted in Fig. 15b. While there is rough 
agreement between the magnitules, there appears no clear correlation with the LFD scaling factor which could be varied by over a factor of two in the experiment. One reaches a similar conclusion after comparing experimental $\tau_{N}$ values with the those computed from Eq. 14 (see Fig. 16).

\section{1\%-D Simulation}

The observed decay of the equilibrium FRC has been modeled with the Los Alamos $1 \%$-dimensional fluid transport code CFRX. Details about the code may be found elsewhere. ${ }^{24,25}$ in brief, CFRX computes the time evolution of the magnetic field, plasma density, electron and ion temperature profiles as a function of the magnetic flux $\psi$. The radial equilibrium and transport equations (i.e., the usual 1-D transport problem) are solved at each time step, subject to four additional constraints imposed by 2-D equilibria: (1) flux-surface averaging within the separatrix; (2) axial contraction (or expansion), consistent with the equilibrium constraints; (3) the average-beta condition, $\langle\beta\rangle=1-1 / 2 x_{s}^{2}$; and (4) parailel conduction and convection between the open-field-line sheath plasma and the end walls. In the code, the resistivity $\eta_{\perp}$ is modeled as a combination of the Spitzer and Bohm values with $\psi$-dependent coefficients. The thermal conductivities $\kappa_{\perp}$ and $\kappa_{\| \mid}$for the electrons and ions are handled in a more limited manner with classical values modified by constant multipliers. These constants, however, may be different in the closed and open field regions. Particle confinement on the open field lines is taken to be the product of the ion free-streaming time, $\tau_{\|}=L / V_{i}$, and a constant multiplier.

A comprehensive set of simulations for all experimental conditions is beyond the intentions of this report. Instead, we have chosen to exhibit resuits from a single run that simulates the "best" conditions on LSM, low-field 3 mtorr with low bias, where the confinement is optimized and most reproducible (see Tables I and II). Initial profiles (see Fig. 17) are chosen to be consistent with the measured parameters just after formation. The ion temperature is chosen to be flat, in view of the fast rates for classical cross-field thermal conduction anticipated for an FRC with $s<2$. The transport coefificients, $\eta_{\perp}, \kappa_{\perp e}$ and $\kappa_{\| e}$, have been varied from their classical values to obtain agreement with the measured parameters at the end of the stable period which is typically $70 \mu \mathrm{s}$ after formation. The profiles at the end of the run are plotted in Fig. 18. As illustrated in Figs. 17 and 18, the resistivity profile is somewhat peaked inside, varying between $11 \times$ classical at the separatrix and $22 \times$ classical at the field 
null. To maintain a relatively flat $T_{\theta}(r)$ profile that is constant in time, the electron thermal conductivities have been adjusted so that $\kappa_{\perp_{\theta}}=35 \times$ classical inside the separatrix and $\kappa_{\|_{\theta}}=0.18 \times$ classical oviside. The anomaly factor for $\kappa_{\perp e}$ is $35 \%$ lower than that obtained in Section IV.B with simple dimensional analysis. Improved confinement on open field lines is evident by the superclassical $\kappa_{\| l}$. Furthermore, as found in earlier simulations of FRX-C data, ${ }^{21}$ the particle confinement time on open field lines is $3 \tau_{\|}$. The average global confinement times predicted by the code, $\tau_{\phi}=160 \mu \mathrm{s}, \tau_{N}=\$ 80 \mu \mathrm{s}$, and $\tau_{E}=74 \mu \mathrm{s}$, are all consistent with the experimental values inferred with the O-D model (cf. Table I).

The LSM simulation is now compared to the one performed for FRX-C using the same $144-D$ code. ${ }^{24}$ For 5-mtorr conditions on FRX-C, the thermal conductivity anomaly factor, $\kappa_{\perp \theta}=36 \times$ classical, was virtually identical to that inferred for 3 mtorr LSM. This finding is somewhat confusing, since the higher $T_{\theta}$ measured on LSM appears to indicate improved confinement. This inconsistency may be resolved by reexamining the FRX-C simulation. In particular, the use of classical parallel electron thermal conductivity on the open field lines cools edge electrons too much, resulting in a low separatrix electron temperature, $T_{e}\left(r_{s}\right) / T_{\theta}(R)=0.6$ (see Fig. 5 of Ref. 24). The temperature gradient in the simulation was significantly larger than that measured on $F R X-C_{0}{ }^{1}$ where $T_{\theta}\left(r_{s}\right) / T_{\theta}(R)=0.86$ (see Fig. 4 of Ref. 1). In the simulation, the large $\mathrm{T}_{\theta}$ gradient, caused by large losses on the open field lines, implies a smaller $\kappa_{10}$ in the closed-field-line region (cf. Eq. 7). From simple dimensional arguments, oire can conclude that the classical anomaly factor $\kappa_{\perp e}$ inferred for FRX-C 5-mtorr conditions in Reference 24 is about three times too low, while the $\kappa_{l l e}$ is too high.

\section{ACKNOWLEDGMENTS}

The author gratefully acknowiedges the persistent encouragement from his Los Alamos colleagues. Special thanks are given to the LSM operations team headed by G. A. Barnes (Los Alamos group CTR-3), to R. E. Chrien (CTR-3) for analyzing the excluded flux and interferometer data and for managing the LSM data base, to K. -M. Ling (CTR-6) for assistance with the 1\%-D transport code, to M. Tuszewski (CTR-3) for useful discussions, and to Prof. Yoshifumi Ito (Osaka University) for a fruitful collaboration during the original design of the Thomson scattering diagnostic for LSM. Technical assistance from R. G. Gribble (CTR-3) and R. Scarberry (CTR5) is deeply appreciated. 


\section{REFERENCES}

1. D. Rej and W. Armstrong, "Electron Temperature Measurements in the FieldReversed Configuration Experiment FRX-C," Nuclear Fusion 24, 177-182 (1984).

2. R. Chrien, K. McKenna, D. Rej, and M. Tuszewski, "Radiation and Impurity Measurements in FRX-C/T," in Proceedings of the 6th US-Japan Workshop Symposium on Compact Toroids, (Hiroshima, Japan, November 1984) K. Watanabe and T. Sato, Eds. (February 1985), pp. 78-82.

3. D. Rej, R. Siemon, and D. Taggart, "The FRX-C/LSM Compression Experiment," in Proceedings of the 9th US Compact Toroid Symposium (LANL preprint L4-UR 89-868, to be published in 1989 by Spectra Technoiogy, Inc., Bellevue, WA).

4. M. Tuszewski, W. Armstrong, R. Bartsch, R. Chrien, et al., "Flux Loss During the Equilibrium Phase of FRCs," The Physics of Fluids 25, 1696-1698 (1982).

5. D. Rej and M. Tuszewski, "A Zero-Dimensional Transport Model for FRCs," in Proceedings of the 5th Symposium on the Physics and Technology of Compact Toroid (Spectra Technology, Inc., Bellevue, WA, 1983) pp. 79-82, and in The Physics of Fluids 27, 1514-1520 (1984).

6. R. Siemon, R. Chrien, W. Hugrass, S. Okada, et al., "FRC Experiments," in Plasma Physics and Controlled Nuclear Fusion Research 1988 (LANL preprint LA-UR 88-2878, to be published by the IAEA, Vienna).

7. C. Choi, R. Chrien, H. Lewis, R. Milroy, et al., "Numerical Examination of HighFlux FRC Equilibria," Bulletin of the American Physical Society 32, 1728 (1987).

8. W. Asmstrong, R. Linford, J. Lipson, D. Platts, and E. Sherwood, "FRC Experiments on Compact Toroids," The Physics of Fluids 24, 2068-2089 (1981).

9. N. Krall, "The Effect of Low Frequency Turbulence on Flux, Particle, and Energy Confinement in an FRC," to be published in The Physics of Fluids.

10. D. Barnes, W. Armstrong, E. Caramana, R. Chrien, et al., "Theoretical and Experimental Studies of FACs," in Plasma Physics and Controlled Nuclear Fusion Research 1986 (IAEA, Vienna, 1987) II, pp. 673-685.

11. K. Freese, R. Bartsch, and R. Siemon, "A Portable Thomson Scattering Apparatus for Use on the Scylla-IVP $\theta-P i n c h, " B u l l e t i n$ of the American Physical Society 23, 848 (1978).

12. R. Chrien, Los Alamos National Laboratory, personal communications (October-November 1988). 
13. M. Tuszewski, "A Semiempirical Formation Model for FRCs," The Physics of Fluids 31, 3754-3759 (1988).

14. R. Spencer, M. Tuszewski, and R. Linford, "Adiabatic Compression of Elongated FRCs," The Physics of Fluids 26, 1564-1568 (1983).

15. R. Siemon, W. Armstrong, D. Barnes, R. Bartsch, et al., "Review of the Los Alamos FRX-C Experiment," Fusion Technology 9, 13-37 (1986).

16. D. Book, "Plasma Formulary," (Naval Research Laboratory. Washington D.C., 1983).

17. S. Hamada, "A Model of Equilibrium Transport and Evolution of Field Reversed Configurations," Nuclear Fusion 26, 729-749 (1986).

18. D. Rej, "Interaction of the Neutral Deuterium Flux with an FRC," in Proceedings of the 6th US Symposium on Compact Toroid Research and the 5th US-Japan Joint Symposium on Compact Toroid Research, M. Yamada and R. Ellis, Jr., Eds. (Princeton Plasma Physics Laboratory, Princeton, NJ, 1984) pp. 214-217.

19. P. Liewer, "Measurements of Microturbulence in Tokamaks and Comparisons with Theories of Turbulence and Anomalous Transport," Nuclear Fusion 25, 543621 (1985).

20. A. Tanga, D. Bartlett, K. Behringer, R. Bickerton, et al., "Experimental Studies in JET With Magnetic Separatrix," op. cit., Ref 10, I, pp. 65-73.

21. J. G. Cordey, D. V. Bartlett, V. Bhatnagar, R. J. Bickerton, et al., "Energy Confinement in JET With Ohmic and Strong Auxiliary Heating," op. cit., Ref 10, I, pp. 99-110.

22. A. Newton, "Electron Temperature in FRCs and $\theta$-Pinches with Closed Magnetic Fields, "Nuclear Fusion 26, 779-783 (1986).

23. D. Correll, "Electron Power Loss Along Open Field Lines," Nuclear Fusion 28, 1075-1083 (1988).

24. K. Werley, "11/-Dimensional Transport Modeling of the FRC," The Physics of Fluids 30, 2129-2138 (1987).

25. M. -Y. Hsiao, K. Werley, and K. -M. Ling, "A 1/4-Dimensional Transport Code for FRC Studies: A User's Guide for CFRX," Los Alamos National Laboratory report LA-11212-MS (May 1988). 
TABLES 
TABLE I

PRESSURE SCAN DATA

\begin{tabular}{|c|c|c|c|c|c|}
\hline PARAMETER & yeits & 2 mtorr & 3 intorr & $4 \mathrm{mion}$ & 5 mtorr \\
\hline fill pressure & mtorr & $2.09 \pm 0.06$ & $3.04 \pm 0.05$ & $4.05 \pm 0.06$ & $4.98+0.04$ \\
\hline bias fieid & $\mathrm{mT}$ & $58 \pm 3$ & $63 \pm 4$ & $57 \pm 4$ & $71 \pm 3$ \\
\hline PI timing & $\mu s$ & $19.2 \pm 0.1$ & $18.6 \pm 0.1$ & $13.9 \pm 0.2$ & $13.9 \pm 0.1$ \\
\hline cusp flux & $m W b$ & $18.8 \pm 0.1$ & $18.8 \pm 0.3$ & $18.8 \pm 0.1$ & $18.8 \pm 0.1$ \\
\hline$B_{w}$ & $\mathbf{T}$ & $0.44 \pm 0.01$ & $0.41 \pm 0.02$ & $0.45 \pm 0.01$ & $0.40 \pm 0.01$ \\
\hline$r_{s}$ & $\mathrm{~mm}$ & $160 \pm 5$ & $151 \pm 12$ & $167 \pm 5$ & $156 \pm 15$ \\
\hline$v_{s}$ & liter & $87 \pm 6$ & $104 \pm 13$ & $115 \pm 7$ & $118 \pm 16$ \\
\hline$\phi_{p}$ & $m W b$ & $4.2 \pm 0.4$ & $3.4 \pm 0.8$ & $5.0 \pm 0.4$ & $3.7 \pm 1.1$ \\
\hline$<n>$ & $10^{20} \mathrm{~m}^{-3}$ & $6.4 \pm 0.4$ & $7.4 \pm 0.6$ & $10.5 \pm 0.8$ & $11.1 \pm 1.1$ \\
\hline$T_{\theta}+T_{i}$ & eV & $670 \pm 40$ & $516 \pm 34$ & $431 \pm 27$ & $328 \pm 27$ \\
\hline$\tau_{\phi}$ & $\mu s$ & $128 \pm 53$ & $158 \pm 36$ & $117 \pm 31$ & $25 \pm 5$ \\
\hline$\tau_{E}$ & $\mu s$ & $54 \pm 11$ & $78 \pm 15$ & $77 \pm 13$ & $23 \pm 5$ \\
\hline$\tau_{N}$ & $\mu s$ & $149 \pm 101$ & $173 \pm 50$ & $160 \pm 79$ & $40 \pm 15$ \\
\hline
\end{tabular}

\section{TABLE \|}

TEMPERATURE vs FILL PRESSURE

$\begin{array}{llllll}\text { PARAMETER } & \text { UNITS } & \text { 2 mtorr } & \underline{3 \text { mtorr }} & \text { 4 mtorr } & \text { 5 mtorr } \\ T_{e}(r=R) & \text { eV } & 165 \pm 15 & 156 \pm 28 & 140 \pm 14 & 142 \pm 15 \\ T_{e}(r=0) & \text { oV } & 133 \pm 24 & 135 \pm 26 & 128 \pm 20 & 117 \pm 18 \\ T_{i}(r \approx R) & \text { oV } & 525 \pm 44 & 368 \pm 37 & 298 \pm 33 & 204 \pm 47\end{array}$




\section{TABLE III}

\section{BIAS SCAN DATA}

\begin{tabular}{|c|c|c|c|c|}
\hline PARAMETER & UNITS & low bins & modium blas & high bias \\
\hline bias field & mT & $44 \pm 5$ & $65 \pm 3$ & $86 \pm 2$ \\
\hline fill pressure & mtorr & $2.99 \pm 0.01$ & $3.04 \pm 0.05$ & $3.03 \pm 0.03$ \\
\hline Pl timing & $\mu s$ & $18.5 \pm 0.1$ & $18.6 \pm 0.1$ & $18.7 \pm 0.1$ \\
\hline cusp flux & $m W b$ & $18.9 \pm 0.1$ & $18.8 \pm 0.1$ & $18.8 \pm 0.0$ \\
\hline$B_{w}$ & $\mathbf{T}$ & $0.39 \pm 0.01$ & $0.41 \pm 0.02$ & $0.41 \pm 0.01$ \\
\hline$r_{s}$ & $\mathrm{~mm}$ & $111 \pm 9$ & $154 \pm 9$ & $186 \pm 6$ \\
\hline$v_{s}$ & liter & $59 \pm 9$ & $107 \pm 10$ & $143 \pm 8$ \\
\hline$\phi_{p}$ & $\mathrm{mWb}$ & $1.1 \pm 0.4$ & $3.5 \pm 0.7$ & $6.3 \pm 0.8$ \\
\hline$\langle n\rangle$ & $10^{20} \mathrm{~m}^{-3}$ & $8.3 \pm 0.6$ & $7.2 \pm 0.6$ & $6.0 \pm 0.5$ \\
\hline$T_{e}+T_{i}$ & $\mathrm{eV}$ & $443 \pm 28$ & $521 \pm 31$ & $582 \pm 34$ \\
\hline$\tau_{\phi}$ & $\boldsymbol{\mu S}$ & $131 \pm 72$ & $160 \pm 34$ & $122 \pm 48$ \\
\hline$\tau_{E}$ & $\mu s$ & $41 \pm 10$ & $82 \pm 14$ & $69 \pm 17$ \\
\hline$\tau_{M}$ & $\mu s$ & $105 \pm 24$ & $185 \pm 53$ & $181 \pm 107$ \\
\hline
\end{tabular}




\section{TABLE N}

\section{EQUILIBRIUM FIELD SCAN DATA}

\begin{tabular}{|c|c|c|c|}
\hline PARAMETES & YNATS & low fifild & high fleld \\
\hline $3_{w} @ 30 \mu s$ & $T$ & $0.41 \pm 0.02$ & $0.57 \pm 0.04$ \\
\hline bias field & $\mathrm{mT}$ & $65 \pm 3$ & $65 \pm 3$ \\
\hline fili pressure & mitorr & $3.04 \pm 0.05$ & $3.05 \pm 0.05$ \\
\hline Pl timing & HS & $18.6 \pm 0.1$ & $18.6 \pm 0.1$ \\
\hline cusp flux & $m W b$ & $18.8 \pm 0.1$ & $20.4 \pm 1.2$ \\
\hline$r_{s}$ & $\mathrm{~mm}$ & $154 \pm 9$ & $130 \pm 8$ \\
\hline$v_{s}$ & liter & $107 \pm 11$ & $69 \pm 8$ \\
\hline$\phi_{p}$ & $m W b$ & $3.5 \pm 0.7$ & $2.8 \pm 0.5$ \\
\hline$\langle n\rangle$ & $10^{20} \mathrm{~m}^{-3}$ & $7.2 \pm 0.6$ & $10.6 \pm 0.9$ \\
\hline$T_{\theta}+T_{i}$ & eV & $521 \pm 31$ & $704 \pm 55$ \\
\hline$\tau_{\phi}$ & $\mu s$ & $160 \pm 34$ & $122 \pm 40$ \\
\hline$\tau_{E}$ & $\mu s$ & $82 \pm 14$ & $57 \pm 7$ \\
\hline$\tau_{N}$ & $\mu s$ & $185 \pm 53$ & $122 \pm 24$ \\
\hline
\end{tabular}




\section{TABLE V}

\section{TEMPERATURE vs EQUILIBRIIJM FIELD}

PARAMETER UNITS

\begin{tabular}{llll}
\hline & & low field & high fleld \\
$T_{e}(r \simeq R)$ & eV & $155 \pm 27^{(a)}$ & $170 \pm 30^{(c)}$ \\
$T_{e}(r=0)$ & eV & $135 \pm 25^{(b)}$ & $155 \pm 32^{(d)}$ \\
$T_{i}(r \simeq R)$ & eV & $368 \pm 37^{(a)}$ & $505 \pm 60^{(c)}$
\end{tabular}

high flekd

low field

(a) ${ }_{B_{w}}(t=30 \mu s)=0.40 \pm 0.01 T$
(b) ${ }_{B_{w}}(t=30 \mu s)=0.41 \pm 0.01 T$
(c) ${ }_{B_{w}}(t=30 \mu s)=0.54 \pm 0.02 T$
(d) ${ }_{B_{w}}(t=30 \mu s)=0.58 \pm 0.03 \mathrm{~T}$ 


\section{TABLE VI}

\section{INPUT POWER TO ELECTRONS}

\begin{tabular}{|c|c|c|c|c|c|c|}
\hline Po & $\mathbf{B}_{\mathbf{b}}$ & $\mathbf{B}_{\mathbf{w}}$ & Equillbration & Compression & Ohmic & Total \\
\hline mtorr & $\underline{\mathrm{mT}}$ & $I$ & eVtus & $\underline{e V / u s}$ & $\mathrm{eV} / \mathrm{ss}$ & $\mathrm{eV} / \mu \mathrm{s}$ \\
\hline 2 & 58 & 0.44 & $2.3 \pm 0.5$ & $0.6 \pm 0.1$ & $0.3 \pm 0.1$ & $3.2 \pm 0.5$ \\
\hline 3 & 53 & 0.41 & $1.7 \pm 0.8$ & $0.3 \pm 0.1$ & $0.2 \pm 0.1$ & $2.2 \pm 0.7$ \\
\hline 3 & 86 & 0.41 & $1.2 \pm 0.8$ & $0.5 \pm 0.1$ & $0.5 \pm 0.1$ & $2.2 \pm 0.8$ \\
\hline 3 & 65 & 0.54 & $3.4 \pm 1.8$ & $0.9 \pm 0.1$ & $0.3 \pm 0.0$ & $4.6 \pm 1.9$ \\
\hline 4 & 57 & 0.45 & $2.0 \pm 0.7$ & $0.3 \pm 0.0$ & $0.3 \pm 0.1$ & $2.6 \pm 0.7$ \\
\hline 5 & 71 & 0.40 & $0.8 \pm 0.7$ & $2.0 \pm 0.5$ & $1.0 \pm 0.2$ & $3.9 \pm 0.8$ \\
\hline & 92 & 0.40 & $1.9 \pm 0.6$ & $1.3 \pm 0.2$ & $1.0 \pm 0.3$ & $4.2 \pm 0.9$ \\
\hline
\end{tabular}




\section{TABLE VII}

\section{ELECTRON POWER FLOW}

\begin{tabular}{|c|c|c|c|c|c|c|}
\hline Po & $\mathbf{B}_{\mathbf{b}}$ & $\mathbf{B}_{\mathbf{w}}$ & $\mathbf{P}_{\mathbf{l n}}$ & $\mathbf{P}_{\mathbf{N e}}$ & $\mathbf{P}_{E_{\mathbf{E}}}$ & $\mathbf{P}_{E E} / P_{\text {tot }}$ \\
\hline mtorr & $\underline{\mathrm{mT}}$ & $I$ & MW & MW & MW & $\%$ \\
\hline 2 & 58 & 0.44 & $43 \pm 8$ & $26 \pm 12$ & $32 \pm 8$ & $21 \pm 6$ \\
\hline 3 & 53 & 0.41 & $40 \pm 13$ & $28 \pm 7$ & $28 \pm 16$ & $26 \pm 16$ \\
\hline 3 & 65 & 0.54 & $80 \pm 35$ & $42 \pm 9$ & $63 \pm 36$ & $29 \pm 12$ \\
\hline 4 & 57 & 0.45 & $75 \pm 17$ & $48 \pm 18$ & $56 \pm 22$ & $38 \pm 16$ \\
\hline
\end{tabular}

\section{TABLE VIII}

ROUGH ESTIMATES OF TRANSPORT COEFFICIENTS

\begin{tabular}{|c|c|c|c|c|c|c|}
\hline $\begin{array}{l}P_{0} \\
\text { mtorr }\end{array}$ & $\begin{array}{l}\mathbf{B}_{\mathbf{b}} \\
\mathrm{mT}\end{array}$ & $\begin{array}{c}\mathbf{B}_{\mathbf{w}} \\
\mathbf{T}\end{array}$ & $\begin{array}{l}x_{\perp} \\
m^{2} s^{-1}\end{array}$ & $\begin{array}{l}k_{\perp} \\
m^{-1} s^{-1}\end{array}$ & $\begin{array}{l}\kappa_{\perp C} \\
m^{-1} s^{-1} \\
\end{array}$ & $\begin{array}{l}\kappa_{\| l c} \\
m^{-1} s^{-1} \\
\end{array}$ \\
\hline 2 & 58 & 0.44 & 250 & $1.6 \times 10^{23}$ & $2.2 \times 10^{21}$ & $4.4 \times 10^{27}$ \\
\hline 3 & 53 & 0.41 & 285 & $2.1 \times 10^{23}$ & $3.9 \times 10^{21}$ & $4.2 \times 10^{27}$ \\
\hline 3 & 65 & 0.54 & 557 & $5.9 \times 10^{23}$ & $5.4 \times 10^{21}$ & $5.5 \times 10^{27}$ \\
\hline 4 & 57 & 0.45 & 714 & $7.5 \times 10^{23}$ & $5.4 \times 10^{21}$ & $3.5 \times 10^{27}$ \\
\hline
\end{tabular}


FIGURES 


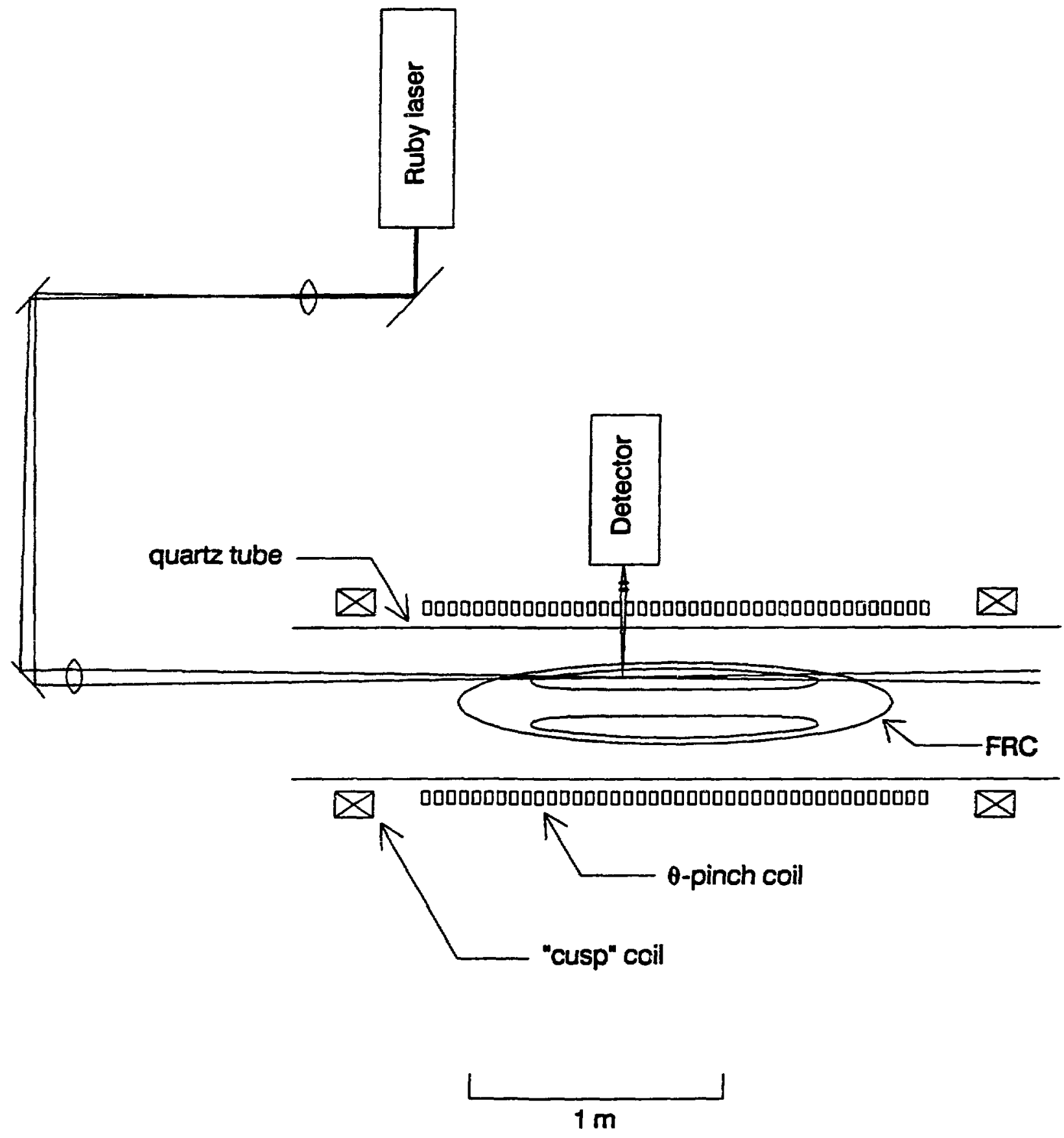

Fig. 1: Schematic diagram of the single-point Thomson scattering electron temperature diagnostic used on FRX-C/LSM. 


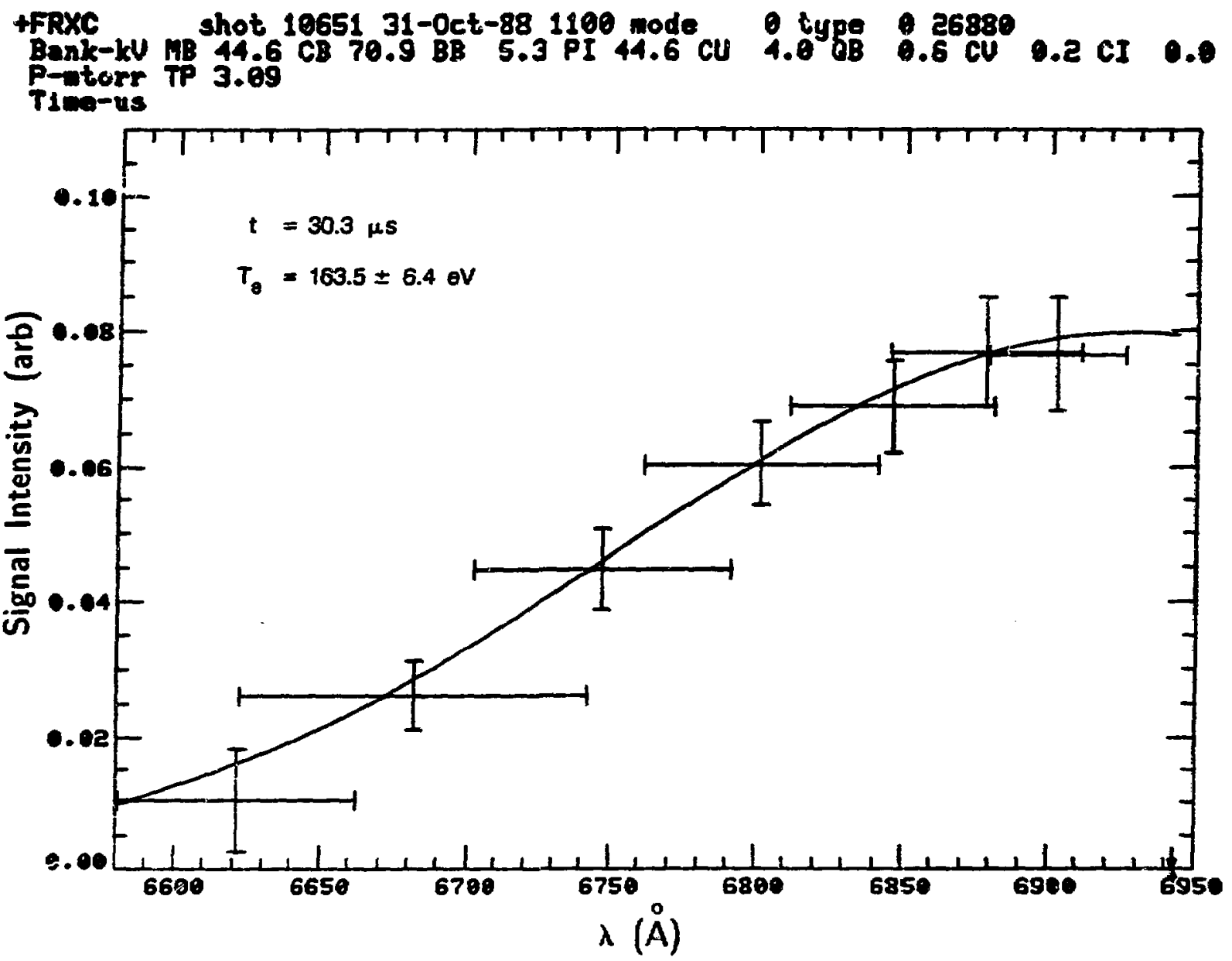

Fig. 2: Thomson scattering data obtained from a single discharge on FRX-C/LSM. 

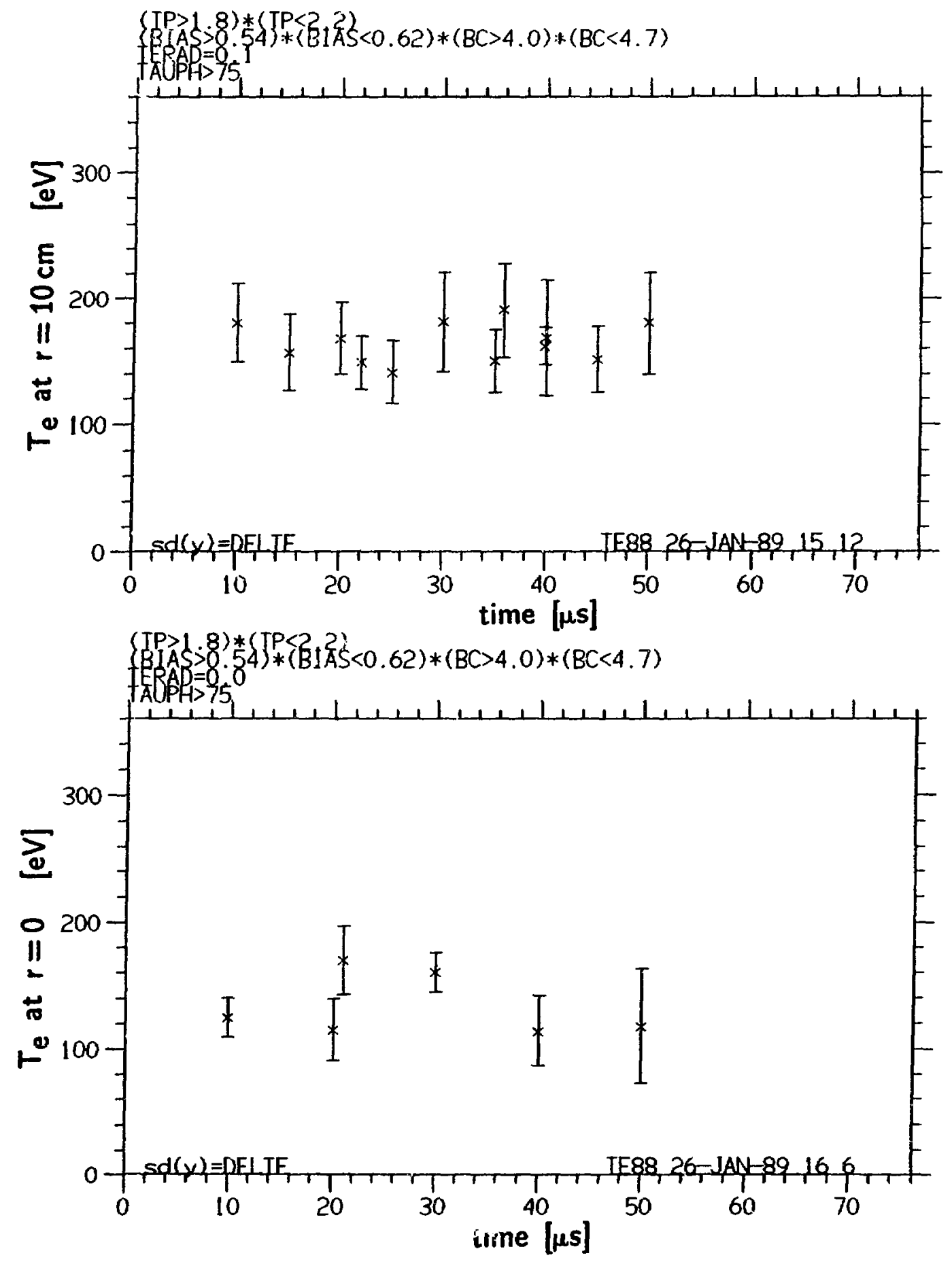

Fig. 3: FRC electron temperature data obtained from $L S M$ at 2 mtorr, low bias, and low external field. 

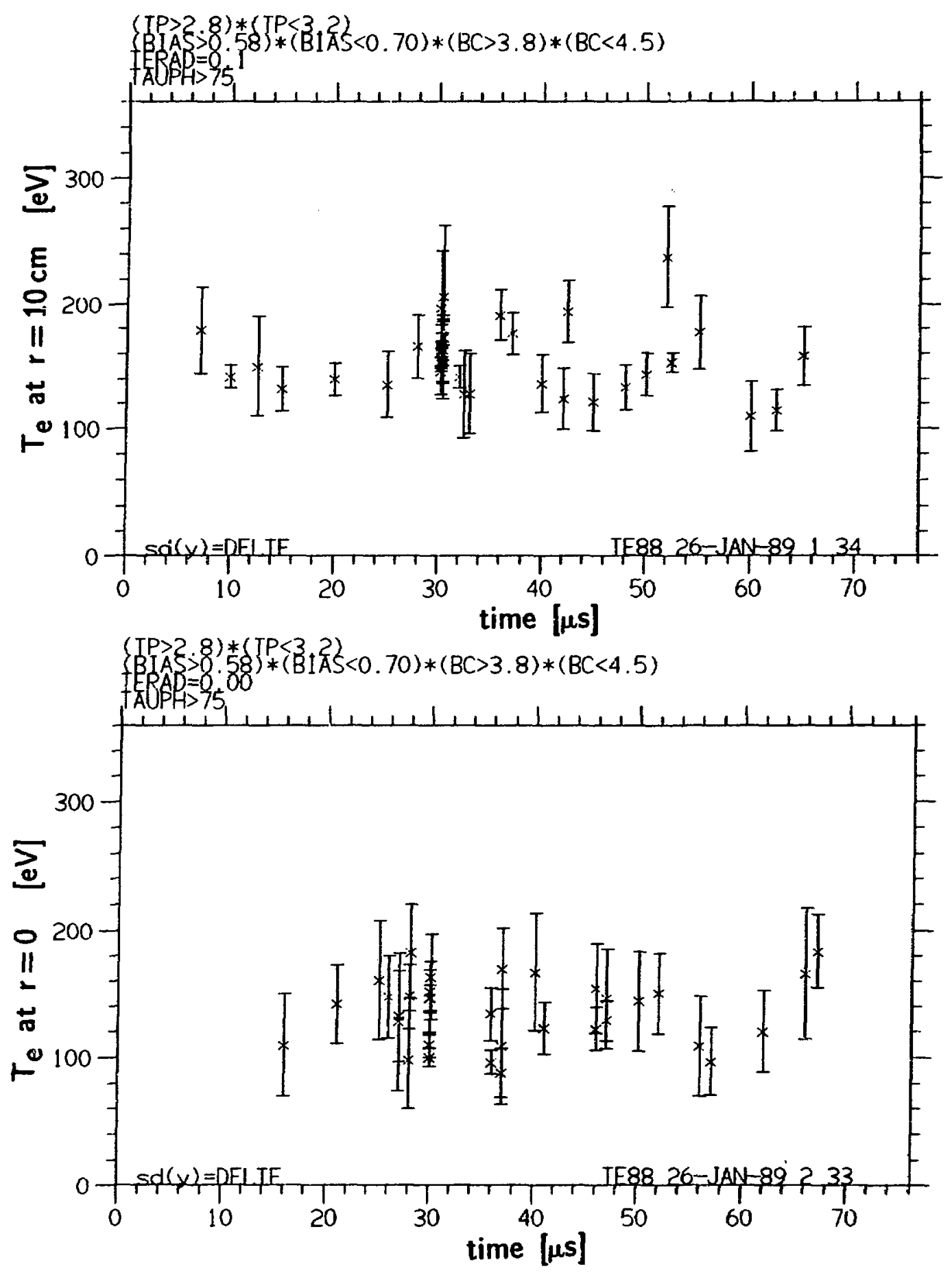

Fig. 4: FAC electron temperature data obtained from $L S M$ at 3 mtorr, low bias, and low external field. 

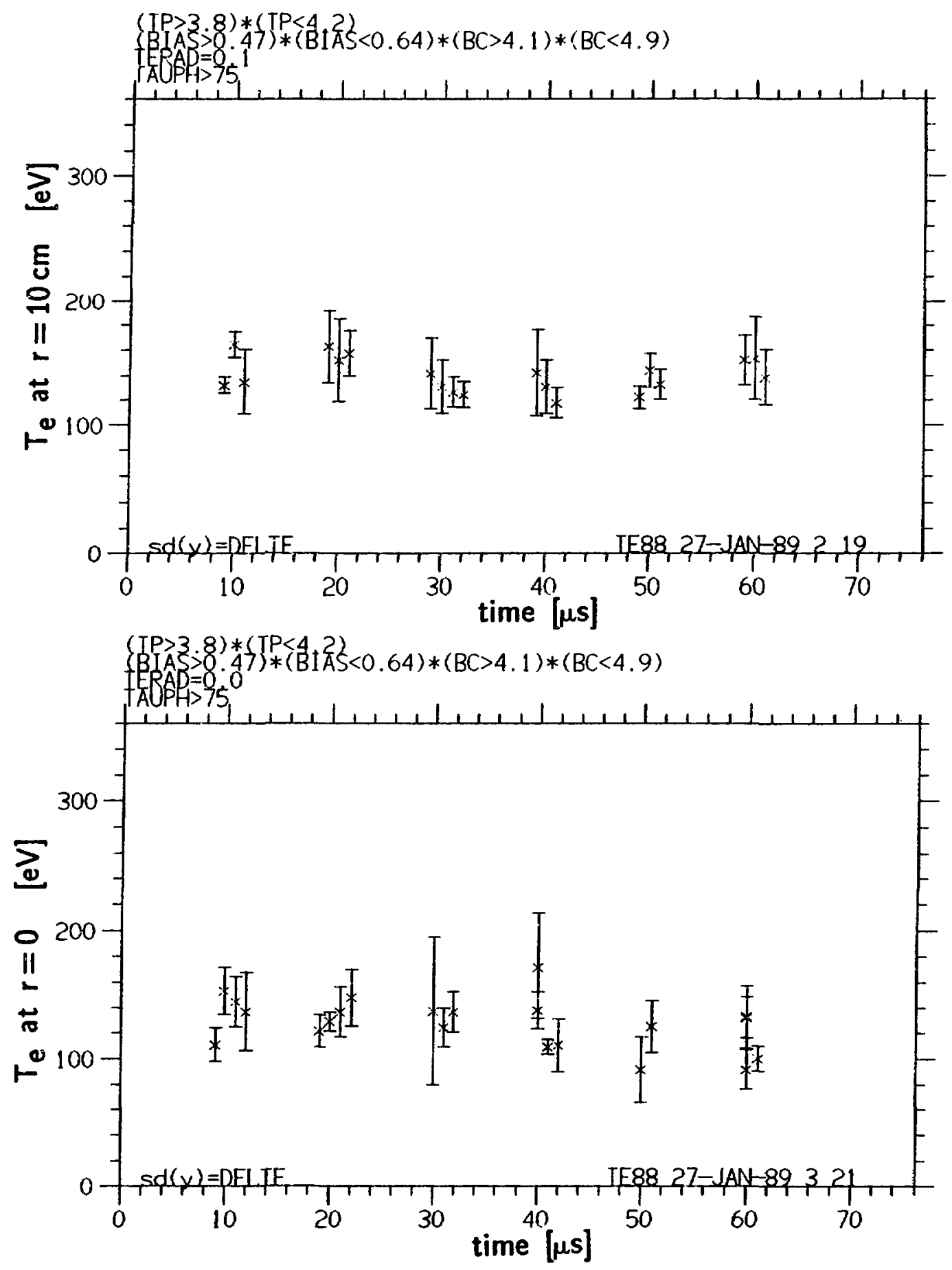

Fig. 5: FRC electron temperature data obtained from LSM at $4 \mathrm{mtorr}$, low bias, and low external field. 

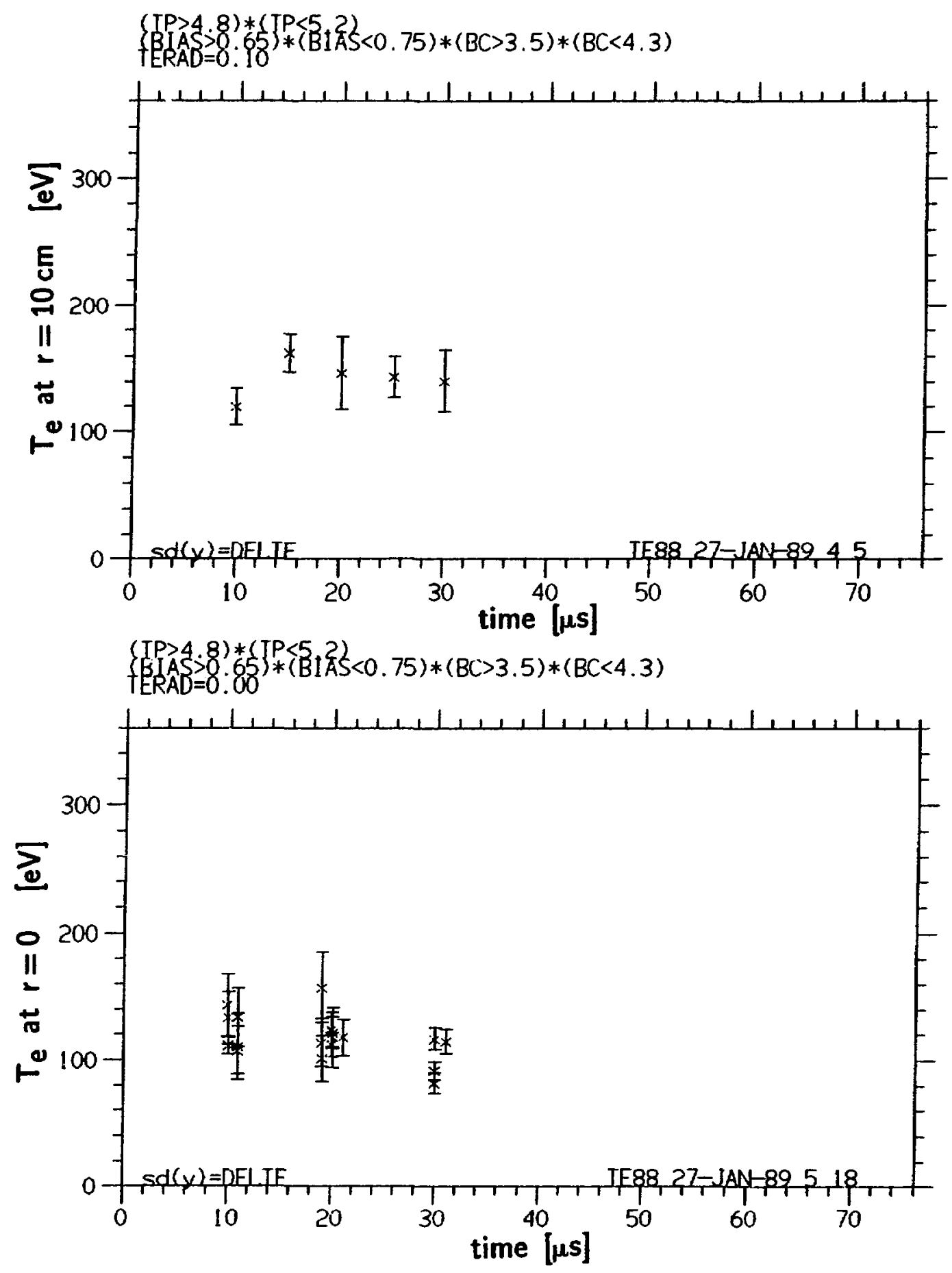

Fig. 6: FRC electron temperature data obtained from LSM at 5 mtorr, low bias, and low external field. 
(a)

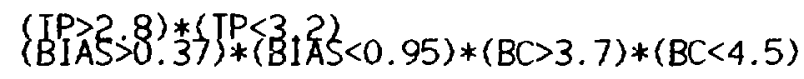

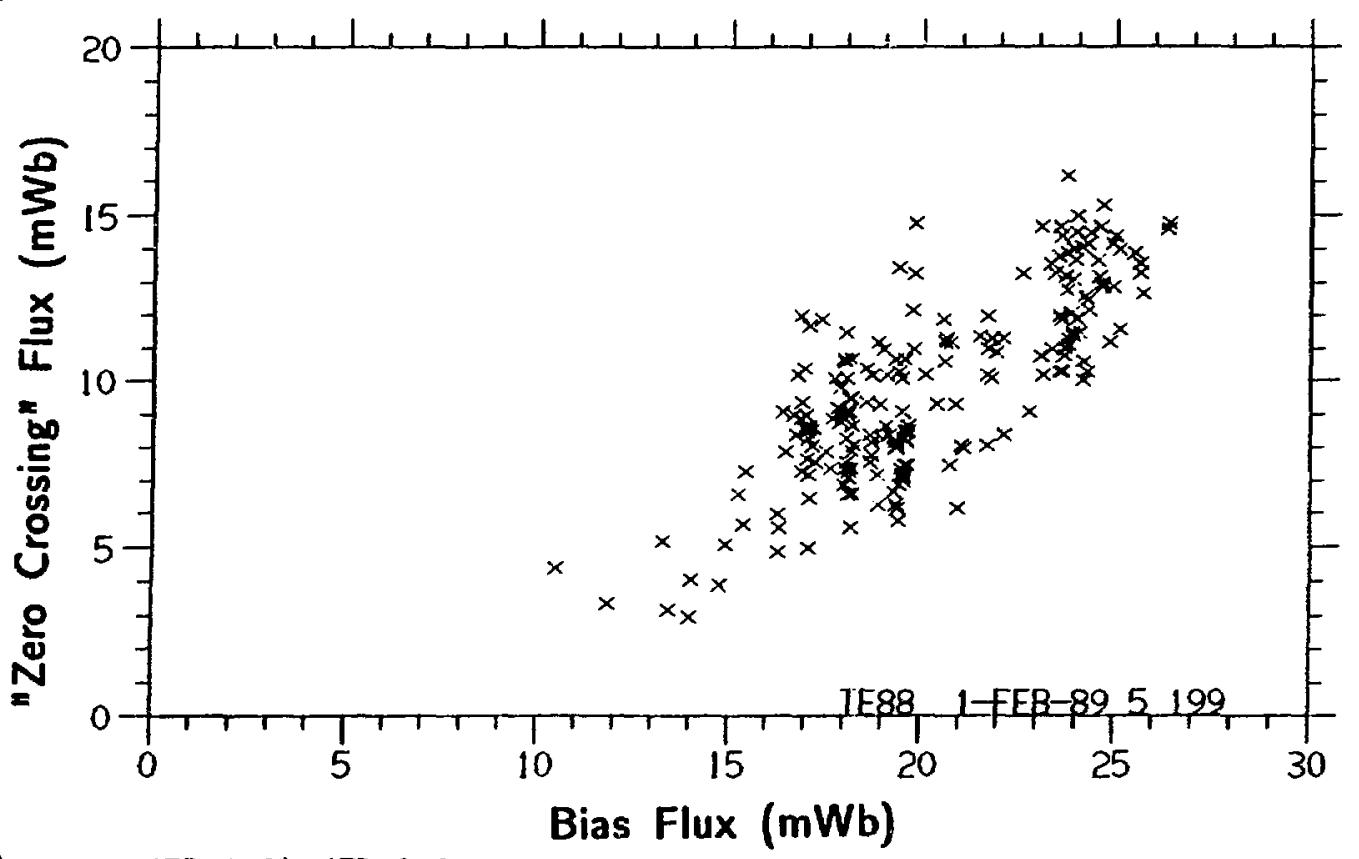

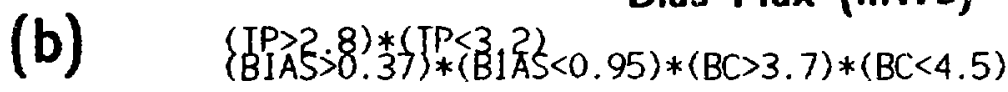

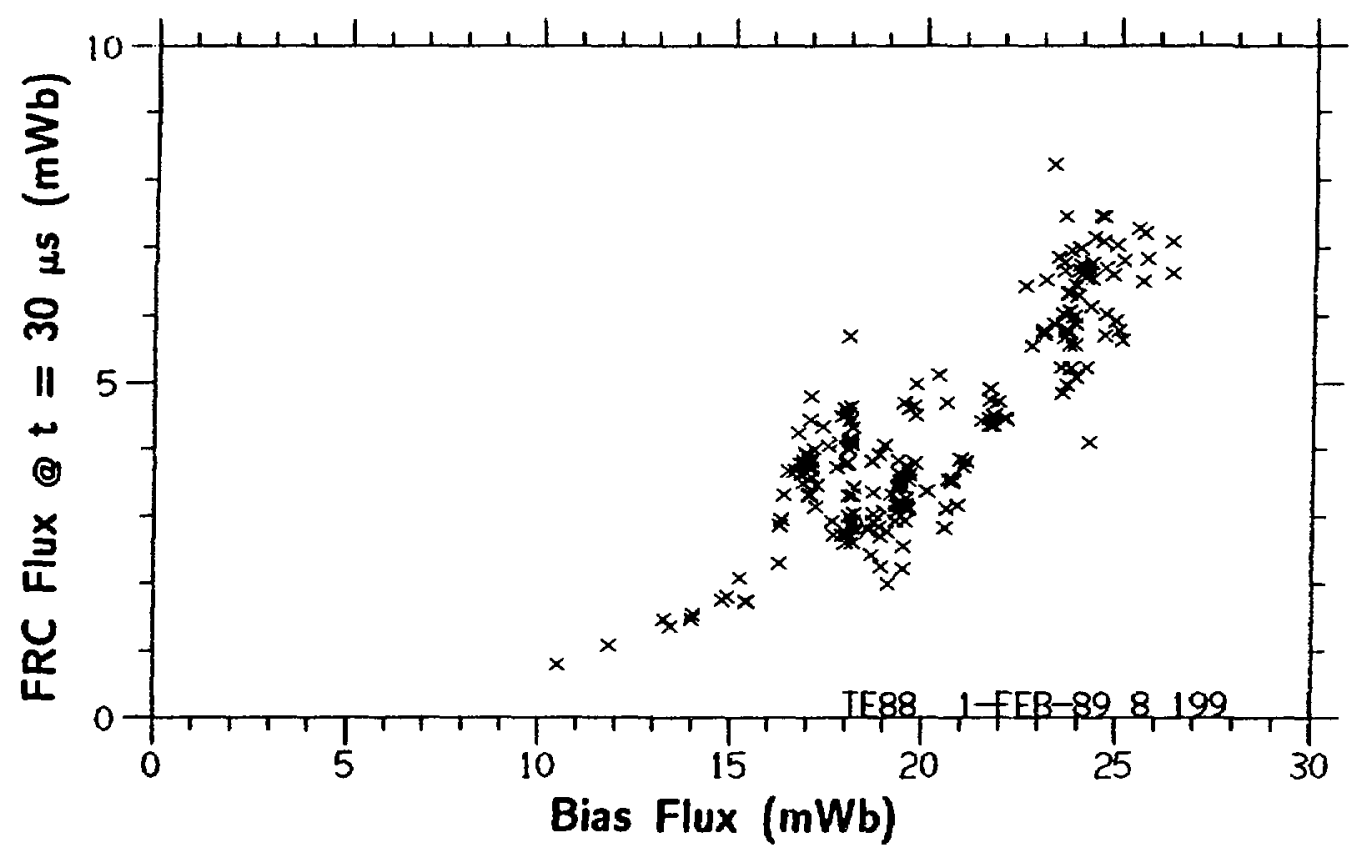

Fig. 7: Poloidal magnetic flux (a) measured at zero crossing and (b) inferred for the equilibrium FRC at time $t=30 \mu$ s plotted $v s$ the initial bias flux for $L S M$ operations at $3 \mathrm{mtorr}$ and low external field. 


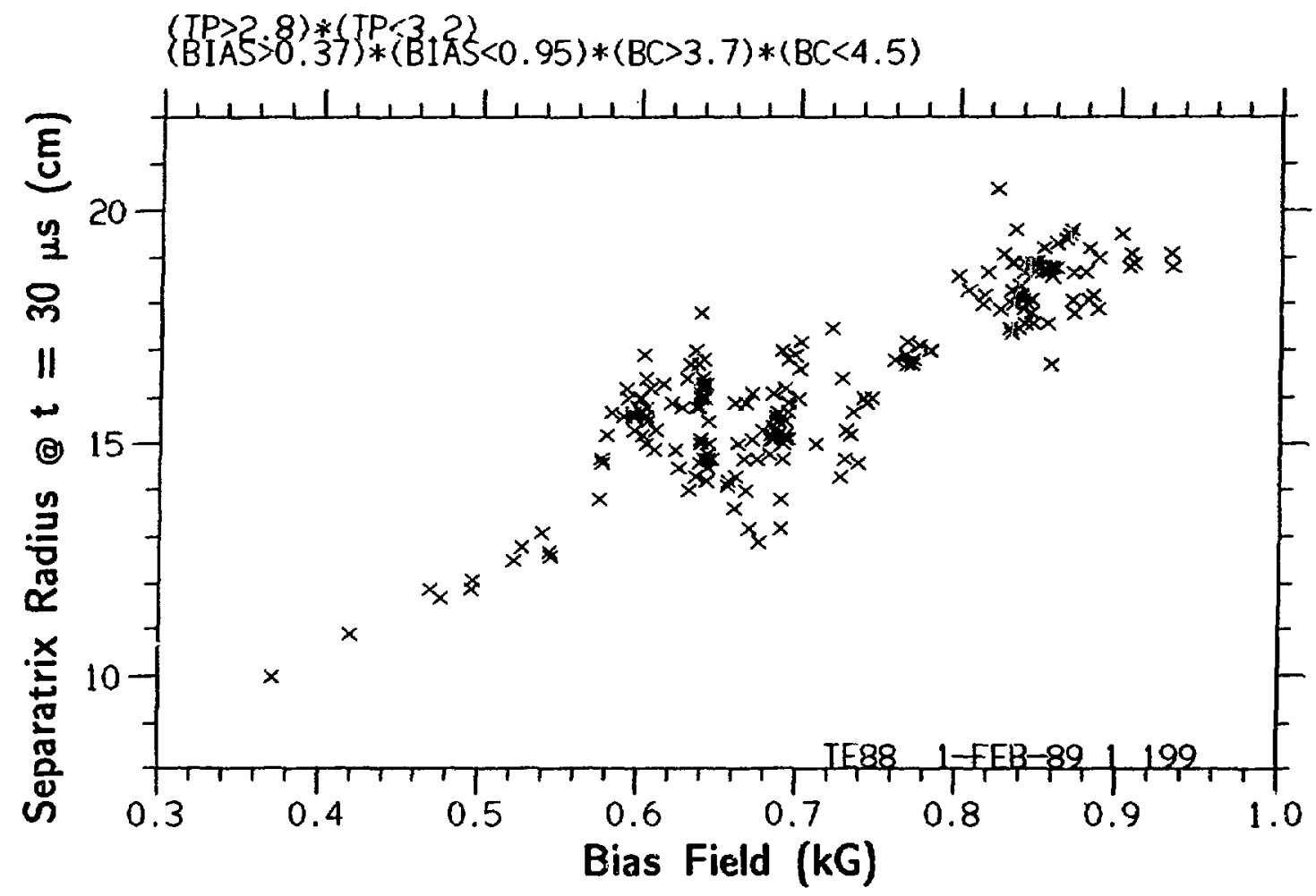

Fig. 8: FRC separatrix radius at $t=30 \mu S$ vs initial bias magnetic field for $L S M$ operations at 3 mtorr and low external field. 

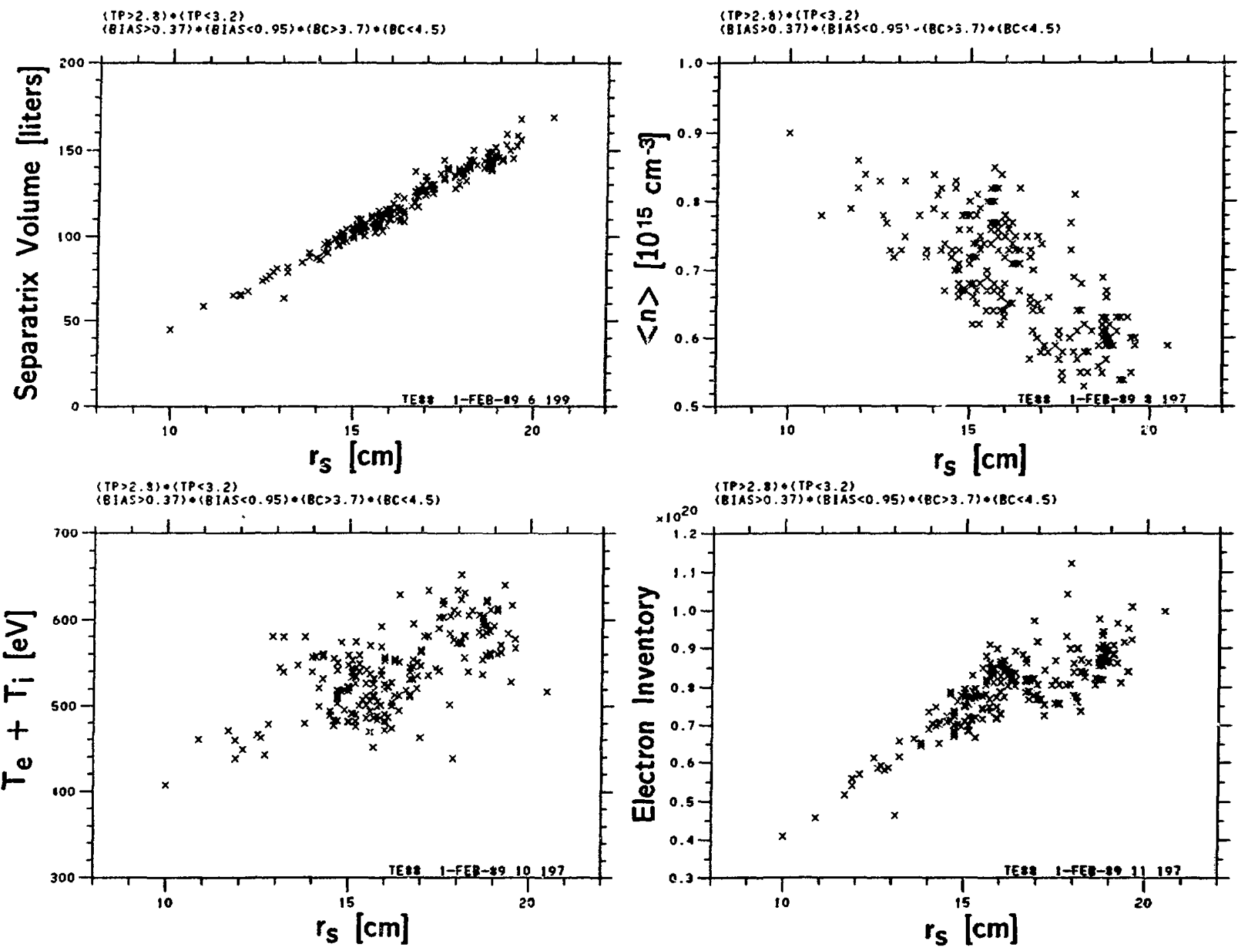

Fig. 9: FRC separatrix volume, average density, pressure balance temperature, and particle inventory at $t=30 \mu, s$ vs separatrix radius measured in $L S M$ during the 3-mtorr bias scan. 

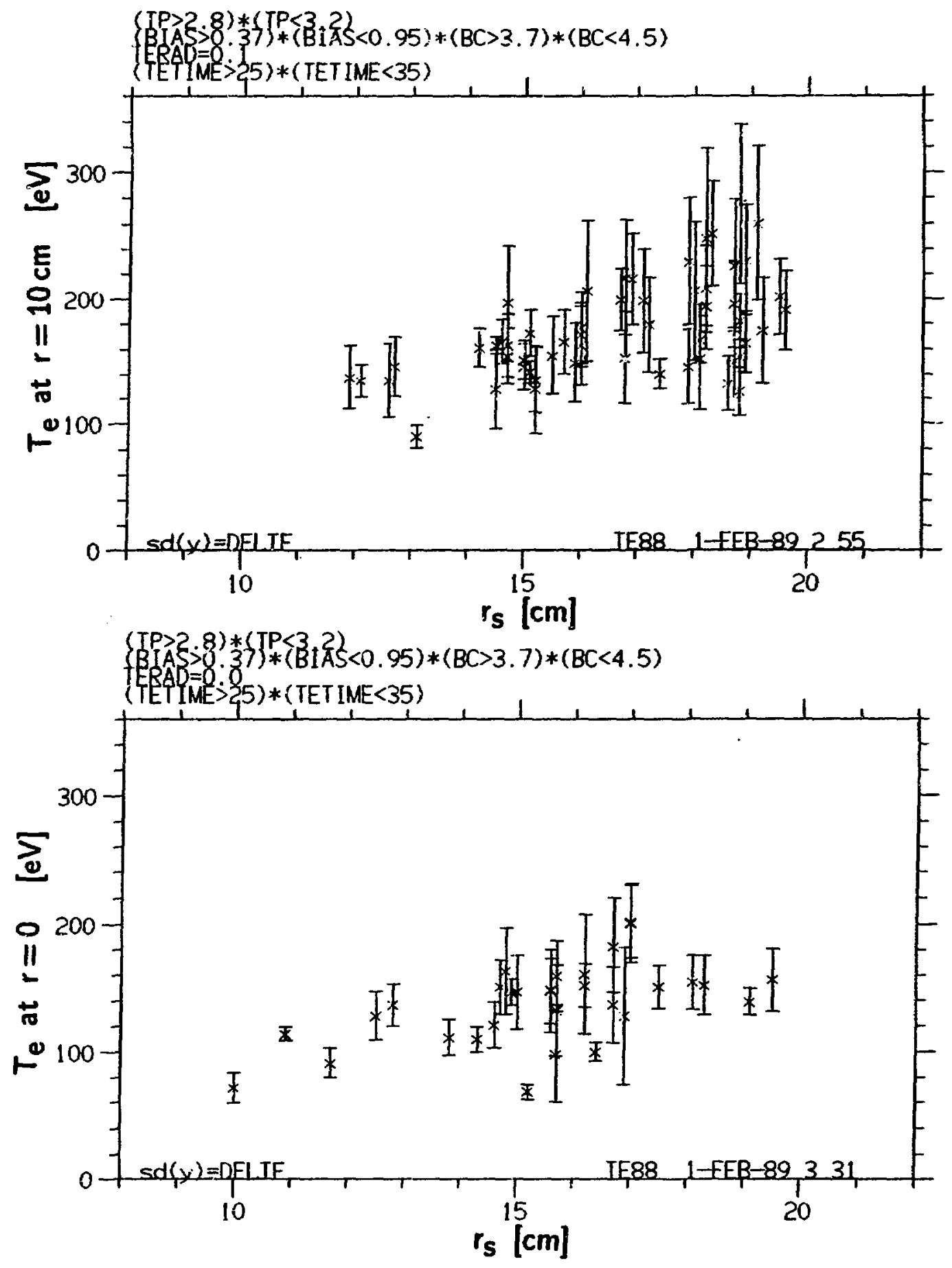

Fig. 10: Electron temperatures at $t=30 \mu s$ vs FRC separatrix radii measured in LSM during the 3-mtorr bias scan. Each data point is from a separate discharge. 

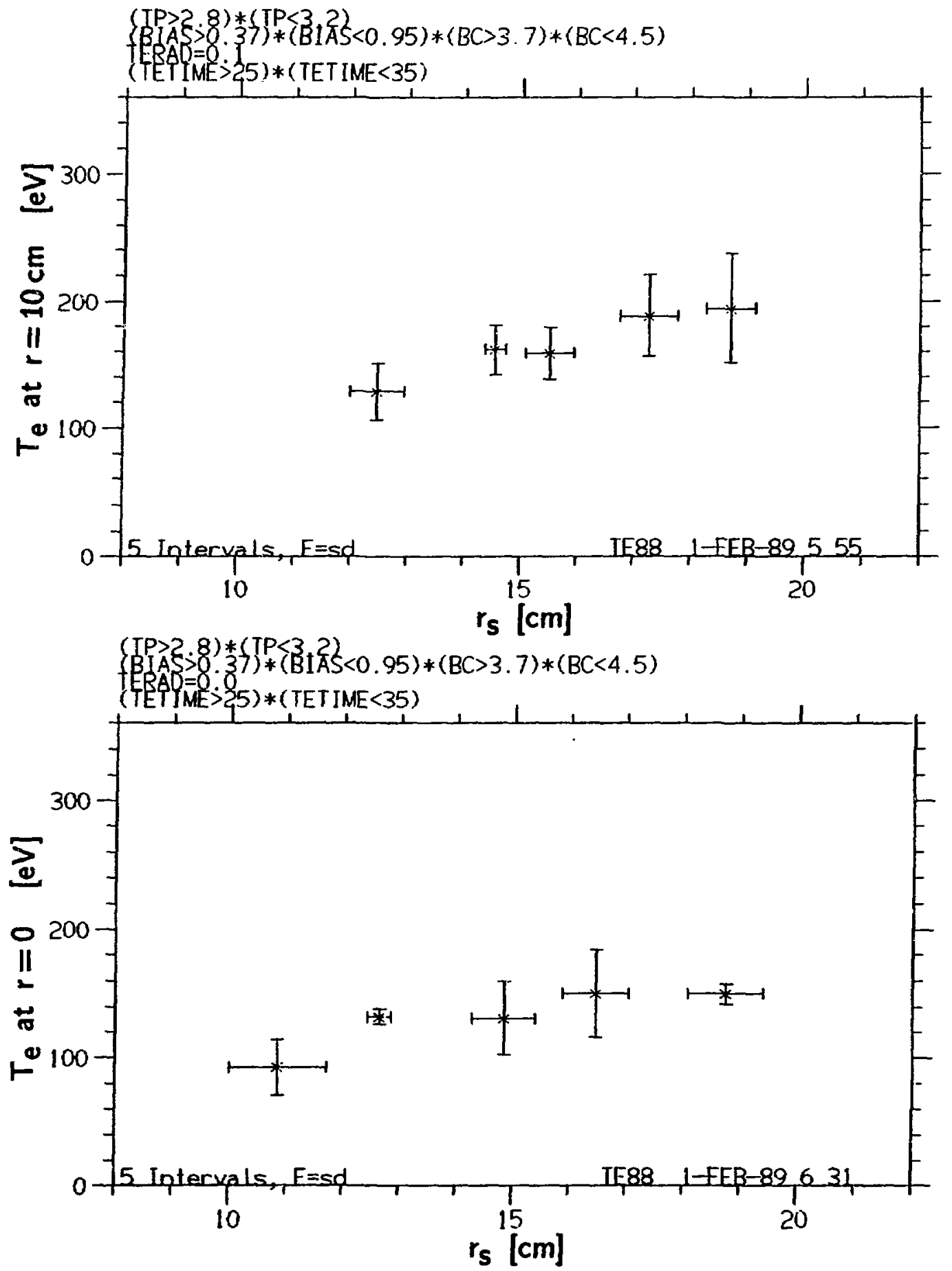

Fig. 11: Electron temperatures at $t=30 \mu \mathrm{s}$ vs FRC separatrix radii measured in LSM during the 3-mtorr bias scan. Data from fig. 10 are averaged into five points with error bars denoting the standard deviations. 

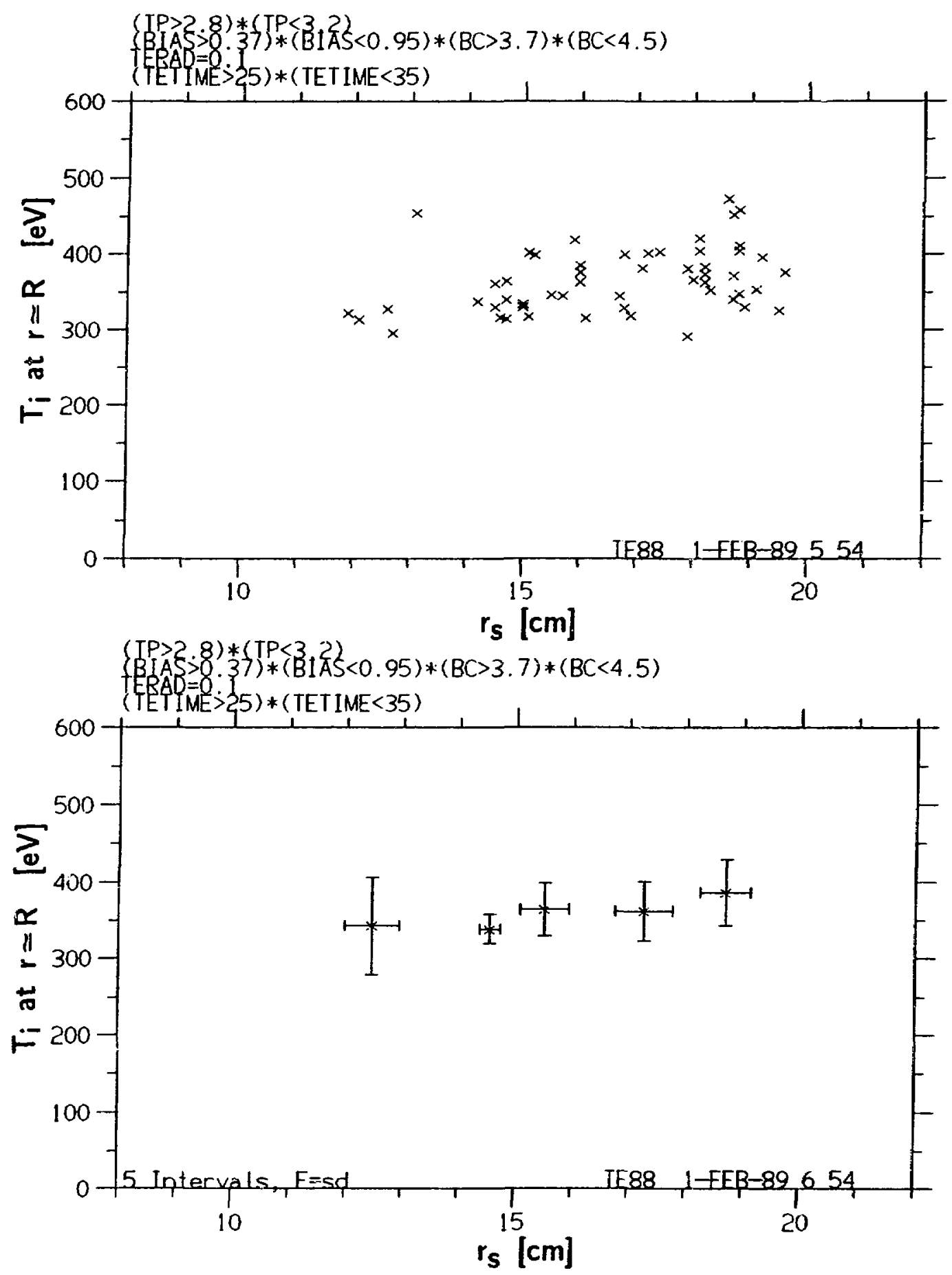

Fig. 12: Inferred field-null ion temperatures at $t=30 \mu \mathrm{s}$ vs FRC separatrix radii obtained in LSM during the 3-mtorr bias scan. Individual data points are plotted in top graph while averages of same data are shown at bottom. 

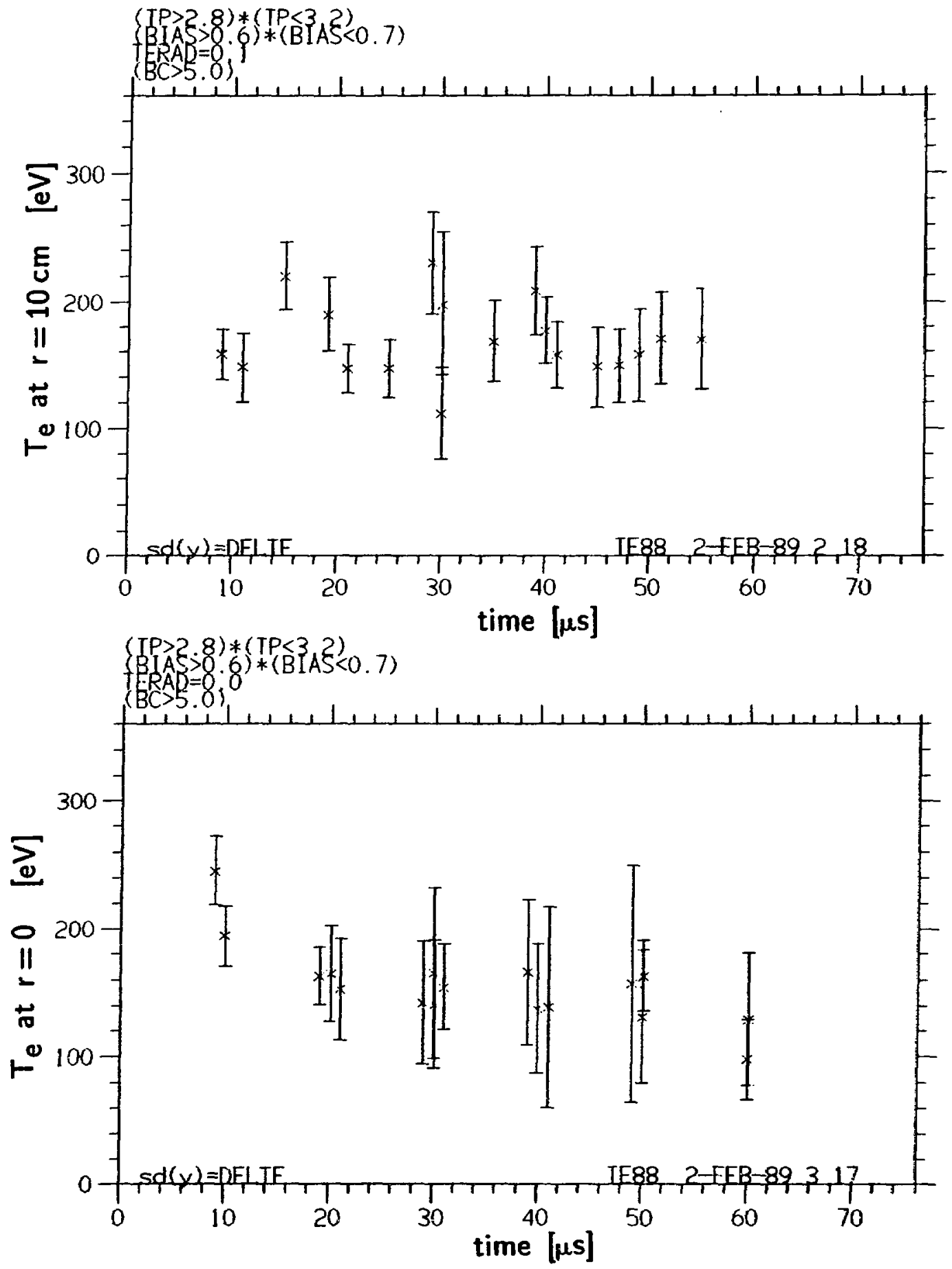

Fig. 13: FRC electron temperature data obtained from LSM at 3 mtorr, low bias, and high external field. 


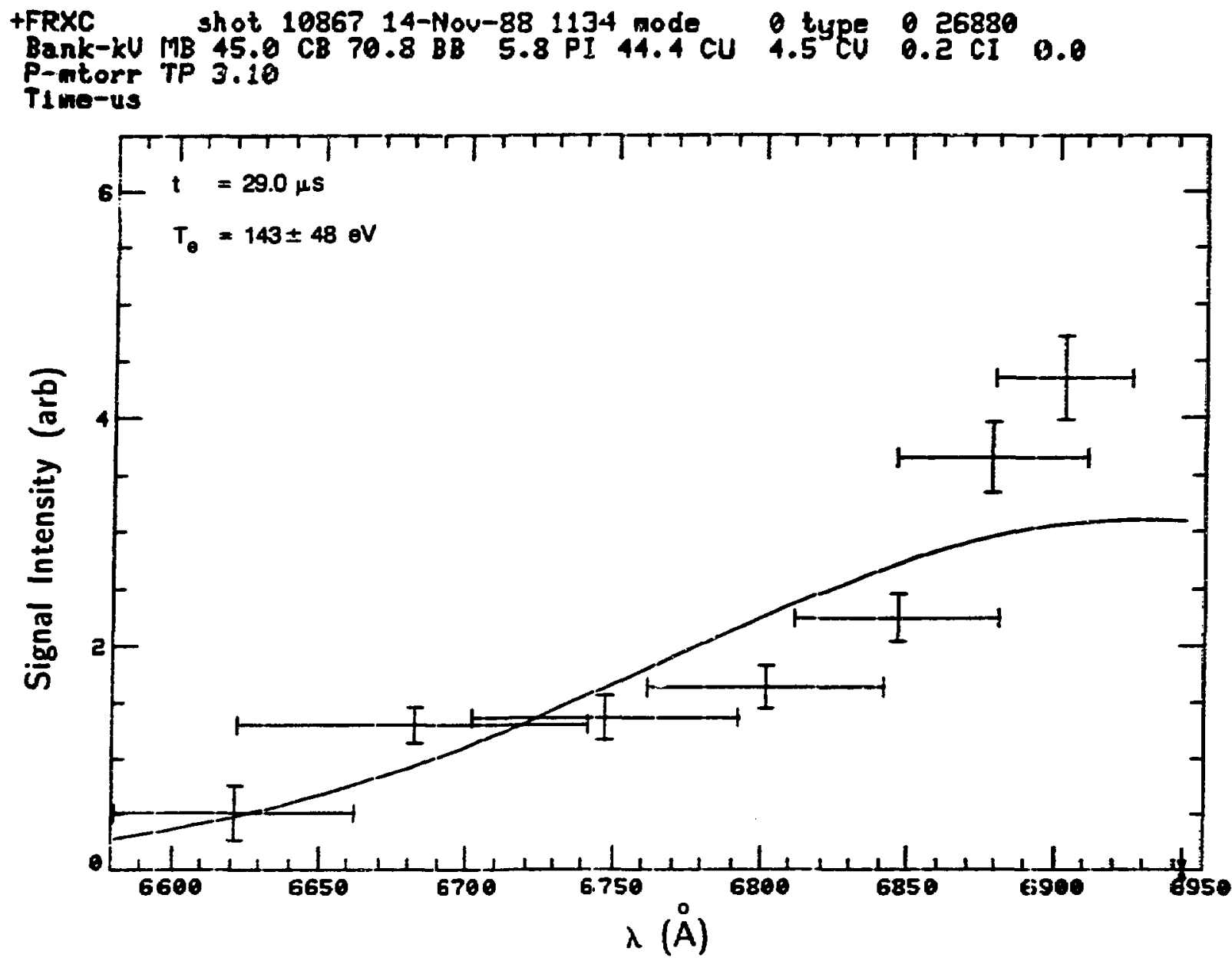

Fig. 14: Representative data obtained from Thomson scattering at the geometric axis on LSM at $3 \mathrm{mtorr}$, low bias, and high external field. Poor singletemperature fits were consistently observed only for this situation. 
(a)
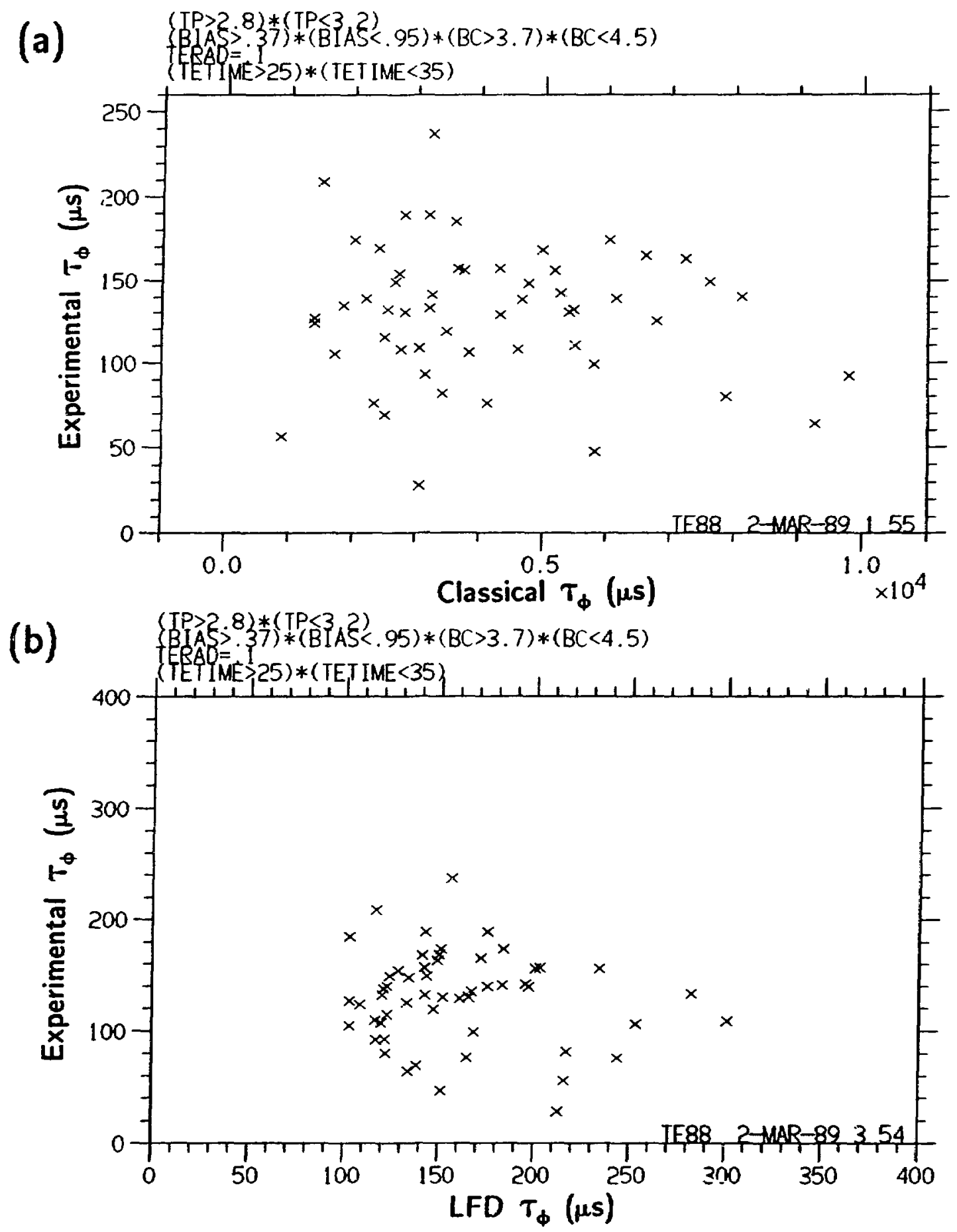

Fig. 15: Experimental flux confinement times plotted vs theoretical predictions from (a) classical and (b) low-frequency-drift instability theory. 


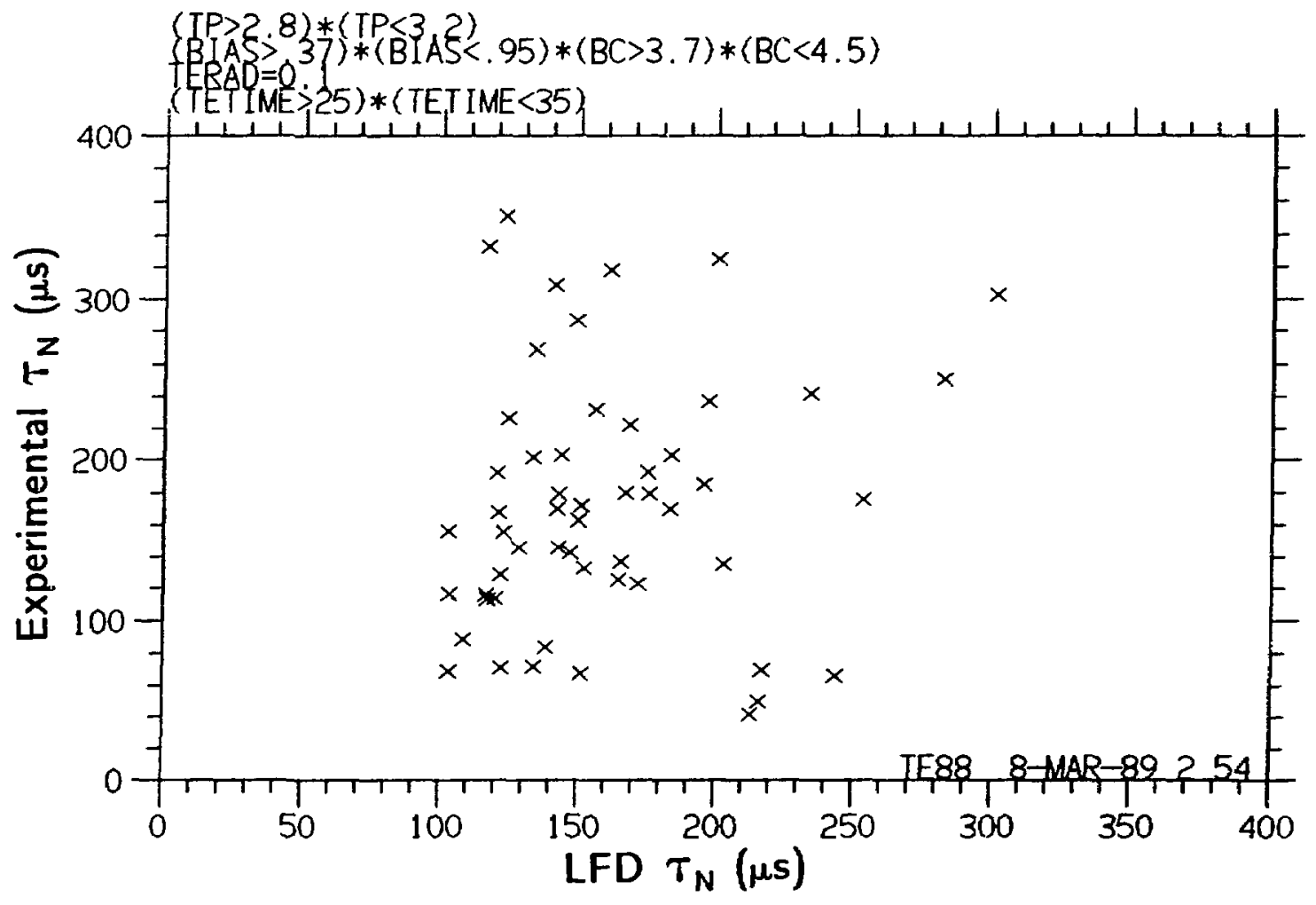

Fig. 16: Experimental particle confinement times plotted against the theoretical predictions from low-frequency-drift instability theory. 

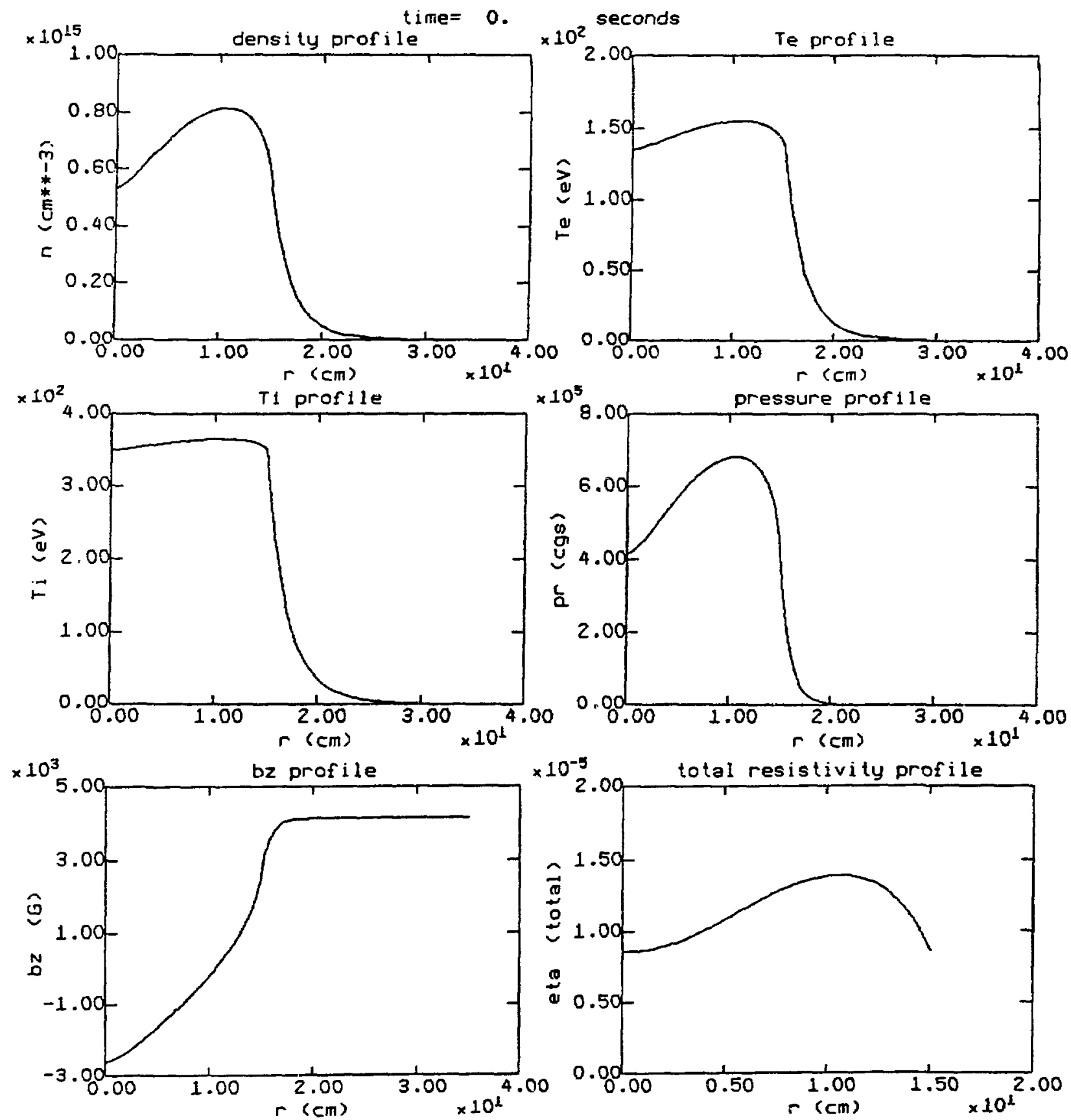

Fig. 17: Initial profiles used in the 1/4-D simulation of an average low-bias, lowfield, 3-mtorr LSM discharge. 

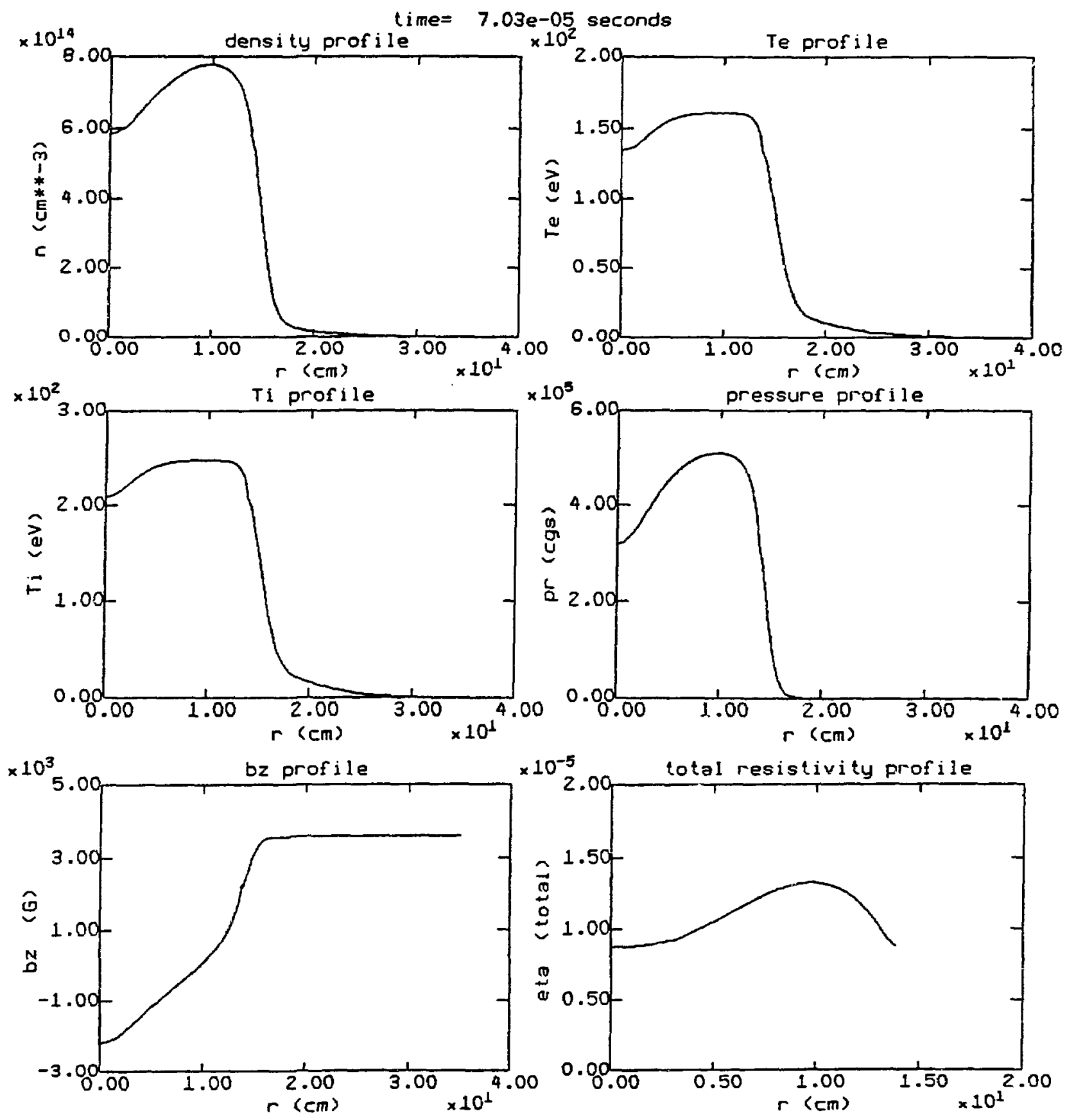

Fig. 18: Final profiles computed at $t=70 \mu \mathrm{s}$ from the $114-\mathrm{D}$ simulation of an average low-bias, low-field, 3-mtorr LSM discharge. 